Research Article

\title{
Fractional-Order Hidden Attractor Based on the Extended Liu System
}

\author{
Yaoyu Wang $\mathbb{D},{ }^{1}$ Ling Liu $\mathbb{D},{ }^{1}$ Xinshan Cai, ${ }^{1}$ Chongxin Liu $\mathbb{D},{ }^{1}$ Yan Wang $\mathbb{D},{ }^{2}$ \\ and Guangchao Zheng $^{3}$ \\ ${ }^{1}$ School of Electrical Engineering, Xi'an Jiaotong University, Xi'an 710049, China
${ }^{2}$ State Grid Wuxi Power Supply Company, Wuxi, Jiangsu 214000, China
${ }^{3}$ Baoding Power Supply Branch of State Grid Hebei Electric Power Supply Co., Ltd., Baoding 071000, China
}

Correspondence should be addressed to Yaoyu Wang; 1641366603@qq.com

Received 23 May 2020; Revised 4 July 2020; Accepted 10 July 2020; Published 4 August 2020

Academic Editor: Ivo Petras

Copyright (c) 2020 Yaoyu Wang et al. This is an open access article distributed under the Creative Commons Attribution License, which permits unrestricted use, distribution, and reproduction in any medium, provided the original work is properly cited.

In this paper, a new commensurate fractional-order chaotic oscillator is presented. The mathematical model with a weak feedback term, which is named hypogenetic flow, is proposed based on the Liu system. And with changing the parameters of the system, the hidden attractor can have no equilibrium points or line equilibrium. What is more interesting is that under the occasion that no equilibrium point can be obtained, the phase trajectory can converge to a minimal field under the lead of some initial conditions, similar to the fixed point. We call it the virtual equilibrium point. On the other hand, when the value of parameters can produce an infinite number of equilibrium points, the line equilibrium points are nonhyperbolic. Moreover than that, there are coexistence attractors, which can present hyperchaos, chaos, period, and virtual equilibrium point. The dynamic characteristics of the system are analyzed, and the parameter estimation is also studied. Then, an electronic circuit implementation of the system is built, which shows the feasibility of the system. At last, for the fractional system with hidden attractors, the finite-time synchronization control of the system is carried out based on the finite-time stability theory of the fractional system. And the effectiveness of the controller is verified by numerical simulation.

\section{Introduction}

In the past few decades, chaos has been established as an important branch of modern physics and mathematics due to its objectivity and universality as well as its wide application, for example, in cryptography [1], economics [2], electronic communication [3], and even in the popular field of neural network $[4,5]$ in recent years. As a pioneer and basic of research, the famous Lorenz system was proposed in 1963 [6]. Based on this, Chen and Ueta system [7] and Lü and Chen system [8] were proposed one after another, which are similar to the Lorenz system but have different topological structure. In 2004, Liu et al. put forward a Liu system which is also a butterfly attractor in [9]. Later, its circuit experimental confirmation was introduced in [10]. With the development of theory and technology, the research has been extended to fractional differential system. Facts show that fractional calculus with a history of more than 300 years is very helpful for interdisciplinary research and has applications in many aspects, such as physics, finance, and sociology. Naturally, the study of the fractional chaotic system has also been reported a lot, such as chaos control and synchronization [11] and fractional memristor [12]. In 2006, Lu and Liu analyzed the dynamics of the fractional Liu system and realized the circuit based on the approximation theory of the fractional operator [13]. In [14], the coupling synchronization of two similar fractional Liu systems is discussed. It is also based on the fractional Liu system, and Hegazi et al. studied the existence and uniqueness of solutions and the influence of the fractional order on chaos control and realized the chaos synchronization of the commensurate fractional Liu system and its integer order counterpart by function projection synchronization in [15]. In [16], a fourth-order commensurate fractional 
hyperchaotic system is proposed by adding a feedback variable to the Liu system, but with 11 terms including three quadratic nonlinearities. In this paper, we propose a new fourth-order commensurate fractional system based on an extension of the three-order Liu system, which is simpler than the system in [16]. It is a particular system with many interesting features that are not available in other systems that have been similarly proposed: (1) it has nine terms, including two quadratic nonlinearities, a weak feedback term, and a constant. (2) It is a hidden hyperchaotic attractor with no equilibrium points. (3) It has small neighborhoods of the origin, and orbit from which is all attracted by the origin, the virtual equilibrium point, despite the fact that its steady-state equations have no real solutions. (4) Although it is four-dimensional, the rank of its Jacobian matrix is less than 4. That is, each row or column of the Jacobian matrix has at least one element that is zero. (5) It is expressed as quaternion fractional differential equations containing a signum function of additional state variable, through which amplitude and polarity of state variables can be transferred to each other, except for the additional state variable. Because the additional state is fed back to another in the form of signum function, whose return is an integer variable, indicating only the sign of the variable. If not all information-containing amplitudes and polarities can be transmitted between variables effectively, the hypogenetic chaotic is defined as a weak feedback chaotic flow with incomplete information transfer [17]. (6) With the constant term selected conditionally, the hidden attractor has nonhyperbolic line equilibrium on the coordinate axis, not no equilibrium points.

The rest of the paper is organized as follows: Section 2 describes some preliminaries of fractional calculus. In Section 3, the new commensurate fractional system is introduced and the dynamical behaviors are numerically analyzed. In Section 4, approximation of the fractional-order integrator and circuit implementation of the new system is discussed. In Section 5, the DSP implementation is introduced. The chaos synchronization controller is designed for the fractional-order system and numerical simulations are carried out in Section 6. Finally, some concluding remarks are drawn in Section 7.

\section{Preliminaries of Fractional Calculus}

Definition 1. Fractional integral of order $\alpha$ for the function can be expressed as follows:

$$
{ }_{a} I_{t}^{\alpha} f(t)={ }_{t} D_{t}^{-\alpha} f(t)=\frac{1}{\Gamma(-\alpha)} \int_{a}^{t} \frac{f(\tau)}{(t-\tau)^{\alpha+1}} \mathrm{~d} \tau,
$$

where $\Gamma$ (.) is the Gamma function, $a$ and $t$ are the bounds of the operation, and $\alpha, a \in R, \alpha>0$. There are many definitions of fractional calculus, and two widely used definitions are the Riemann-Liouville definition and Caputo definition. For the reason that the initial values of the Caputo fractional-order differential equations are clear, it is more common in engineering applications.

Definition 2. The Caputo definition of fractional derivatives can be written as

$$
{ }_{a}^{C} D_{t}^{\alpha} f(t)=\frac{1}{\Gamma(n-\alpha)} \int_{a}^{t} \frac{f^{(n)}(\tau)}{(t-\tau)^{(\alpha-n+1)}} \mathrm{d} \tau,
$$

where $n-1<\alpha<n$, and the $C$ represents the Caputo definition.

\section{System Model and Dynamics Analysis}

In this section, we first present the Liu system given by Liu et al. [9]:

$$
\left\{\begin{array}{l}
\dot{x}=a(y-x), \\
\dot{y}=b x-k x z, \\
\dot{z}=-c z+h x^{2} .
\end{array}\right.
$$

In which, $x, y$, and $z \in R$ are the states and $a, b, c, k$, and $h \in R$ are parameters. By simulation, it can be easily come out that system (3) is chaotic with $a=10, b=40, c=2.5, k=1$, and $h=4$ [9]. Hence, a 4D commensurate fractional-order system is generated from system (3) as follows:

$$
\left\{\begin{array}{l}
\frac{\mathrm{d}^{q} x}{\mathrm{~d} t^{q}}=a(y-x), \\
\frac{\mathrm{d}^{q} y}{\mathrm{~d} t^{q}}=b x-k x z+d \operatorname{sign}(w)+m, \\
\frac{\mathrm{d}^{q} z}{\mathrm{~d} t^{q}}=-c z+h x^{2}, \\
\frac{\mathrm{d}^{q} w}{\mathrm{~d} t^{q}}=-g y,
\end{array}\right.
$$

with the same values of $(a, b, c, k$, and $h)$, and settle $g=5$ and and $d=20$. When $m=20$, the equilibrium point of system (4) is in the negative half of the $w$-axis, and the hidden attractor is shown in Figure 1 with final time 3600 and a time step of 0.005 .

Comparing with the original, a weak feedback term about $w$ is the feedback to the second equation of the system in the form of a symbolic function. Then, the steady-state equations can be written as

$$
\left\{\begin{array}{l}
a(y-x)=0, \\
b x-k x z+d \operatorname{sign}(w)+m=0, \\
-c z+h x^{2}=0, \\
-g y=0 .
\end{array}\right.
$$

If $m=-20$, the equilibrium point of system (4) is in the positive half of the $w$-axis. Then, a system with line equilibrium can be obtained. The line equilibrium is located on the $w$-axis except for the origin. To estimate the Jacobian matrix, the partial derivatives of the state variables are required. For calculation, the sign $(w)$ is replaced by the tanh (nw), where $n$ is a large positive number. It can be seen from Figure 2 that the larger the $n$ is, the more similar the graph of the function tanh (nw) is to the graph of the signum function. 


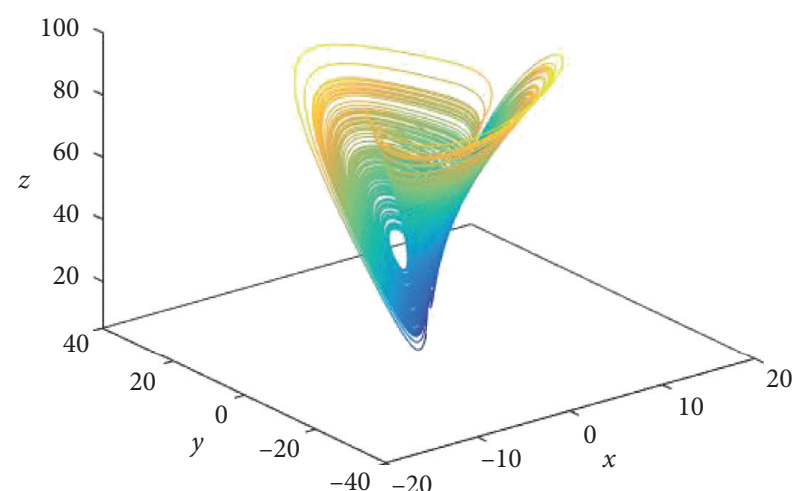

(a)

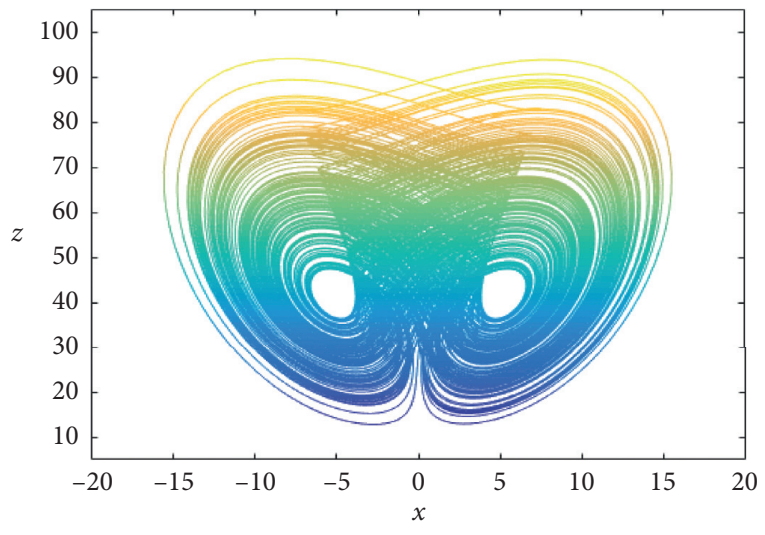

(c)

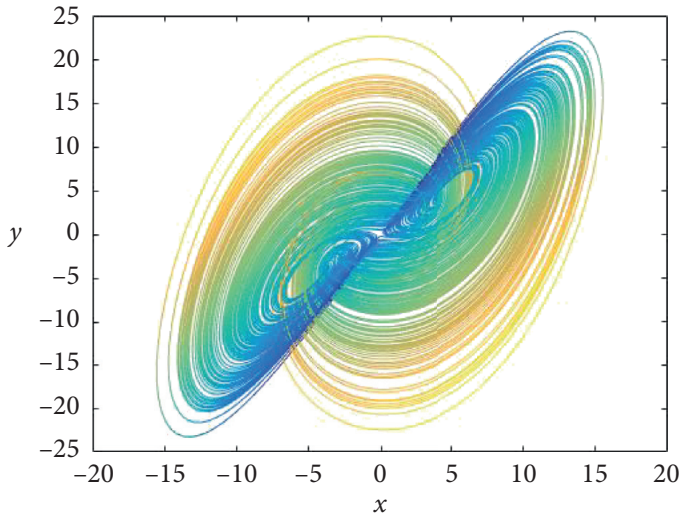

(b)

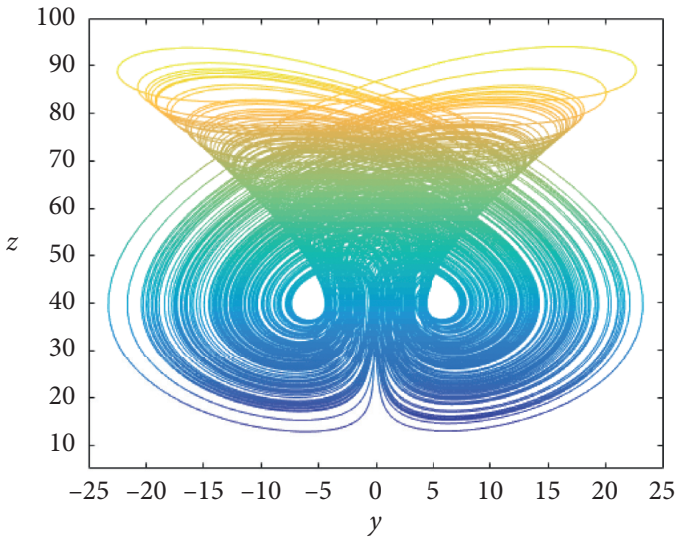

(d)

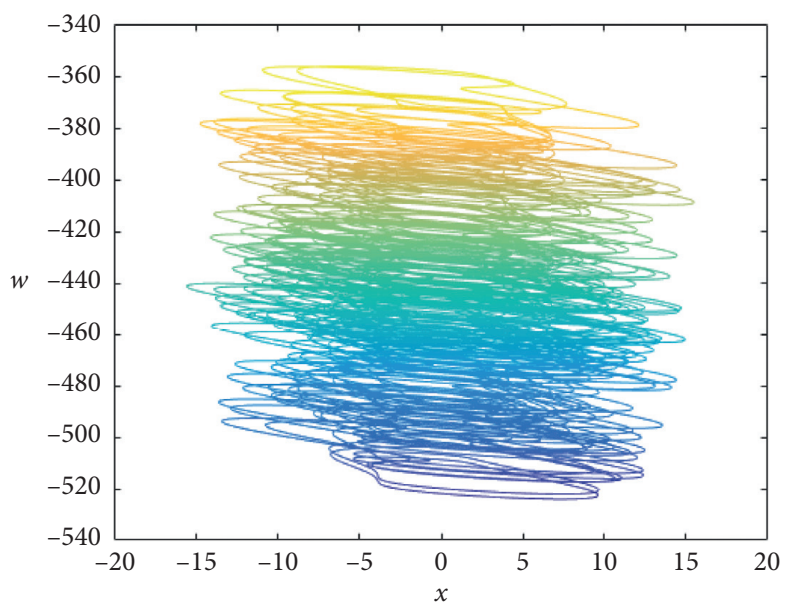

(e)

Figure 1: The chaotic system with line equilibrium points for $a=10, b=40, c=2.5, k=1, h=4, g=5, d=20$, and $m=20 ; X(0)=(2.2,2.4,0.8$, 0 ): (a) in the $x-y-z$ space, (b) on the $x-y$ plane, (c) on the $x-z$ plane, (d) on the $y-z$ plane, and (e) on the $x-w$ plane.

Via linearizing, the Jacobian matrix can be gotten as

$$
J=\left[\begin{array}{cccc}
-\mathrm{a} & a & 0 & 0 \\
b-k z & 0 & -k x & \mathrm{~d} n\left(1-\tanh ^{2}(n w)\right) \\
2 h x & 0 & -c & 0 \\
0 & -g & 0 & 0
\end{array}\right] .
$$

And the eigenvalues are calculated as for $w \neq 0, \lambda_{1}=0, \lambda_{2}=15.6155, \lambda_{3}=-2.5, \lambda_{4}=-25.615$.

Hence, regardless of the value of $m$, there is an eigenvalue whose real part is zero. A nonhyperbolic equilibrium point has one or more eigenvalues with a zero real part. Therefore, the system has a nonhyperbolic line equilibrium which is uncommon in chaotic systems with hidden attractors. 


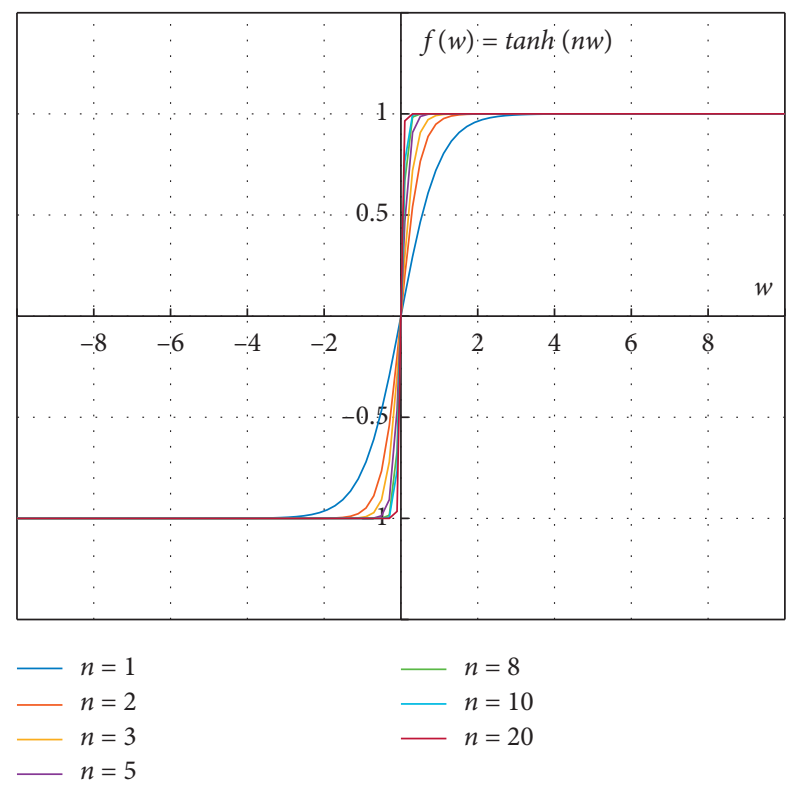

Figure 2: The graph of the function $f(w)=\tanh (\mathrm{nw})$.

On the other hand, when $m \neq 20$, there are three results in the steady-state equations $x_{e}=y_{e}=z_{e}=0$. However, there is no result for $w_{e}$, which signifies that system (4) has no equilibrium. And the hidden attractor can be seen in Figure 3 with $m=1$ and $q=0.99$.

When there is no equilibrium, the 3D Poincare mapping is also plotted in Figure 4, which also illustrates the chaotic characteristics of the system (4). The plane we chose is $x+w=0$. Then, in order to better analyze the trend of the trajectories, we mark them with different colors according to the different directions of the trajectories. If phase trajectories of system (4) cross the plane in a positive direction $(x+w>0)$, the trace will be painted in blue. If phase trajectories of system $(4)$ cross the plane negatively $(x+w<0)$, the trace will be painted in orange. The scattered points shown in Figure 4 are consistent with the characteristics of chaos.

3.1. The Influence of Parameters on the System. Proverbially, bifurcation diagram and the LE are two dynamical tools to investigate the dynamics behaviors of nonlinear systems. Through investigating the bifurcation with respect to changing parameters, the dynamic behaviors of the system can be explored. While the LE not only can exhibit the projections of the system but also can display the changes for each state variable.

The calculated values of the LEs of system (4) considering various sampling and observation time with $m=1$ and $m=20$ are given in Table 1 and 2, respectively. Here, the hyperbolic tangent function is still utilized and the Jacobian matrix used in the calculation is shown in equation (6).

It is clear from Table 1 and Table 2 that under different parameter values, observation time, and sampling time, the hidden attractor has a $(+,+, 0,-)$ sign of Lyapunov exponent demonstrating a 4D hyperchaotic system [18]. Moreover, the
Lyapunov exponents for $m=1$ and $m=20$ are shown in Figures 5 and 6, respectively. It is obvious from Figures 5 and 6 that system $(4)$ with has $(+,+, 0,-)$ nature of the Lyapunov exponents and thus confirm hyperchaotic nature of system (4).

With the change of the parameter $m$, the bifurcation and LE of system (4) are shown in Figures 7(a) and 7(b). System (4) displays abundant dynamic behavior when changing the value of the bifurcation parameter $m$ from 45 to 70 . As can be seen in Figure $7(\mathrm{~b})$, there is a reverse period-doubling route to chaos, when increasing the value of the parameter $m$ from 45 to 53.5 , except for $47.5<m<48.25$, and the chaotic behavior is shown. In addition, when the parameter $m>53.5$, system (4) exhibits a series of evident perioddoubling bifurcations. When the control parameter $m$ is increased above the value of 68.75 , the attractor enters periodic behavior eventually.

For fractional system models, the behavior of the system varies greatly at different orders. The bifurcation with $q$ is shown in Figure 8, from which chaos and periodic behavior can be clearly observed. And the chaotic behavior can still be observed when $q=0.835$, illustrating that the chaotic characteristics of the proposed system are significant. Besides, when in the section of $(0.877,0.887)$ and $(0.937,0.96)$, the system is periodic. However, when $q>0.975$, chaos appeared again. These characteristics are confirmed again by the phase plane plot. In Figure 9 with changing the $q$, the period (as Figure 9(a)), period-doubling (as Figure 9(b)), and chaotic (as Figure 9(c)) trajectories are shown, which are consistent with the conclusion and analysis in Figure 8.

Furthermore, the largest Lyapunov exponents (LEmax) for two parameters are calculated in Figure 10. In Figure 10(a), the maximum of the LEmax can exceed 2, which indicates the chaotic system. However, in some parameter values, the LEmax can be less than 0, and there may be periodic trajectories or a stable case. Figure 10(b) is about parameters $c$ and $h$. The LEmax shows obvious symmetry about the value of $h$. In Figure 10, as the LEmax increases, the color changes from dark blue to yellow. Hence, for the selection of parameters, the parameters in the dark blue region corresponding to the LEmax equal to or less than 0 are more likely to lead to limit cycle or stability. In the light green region corresponding to the positive LEmax, it can be considered as weak chaos. The yellow region corresponding to a large positive LEmax denotes chaotic motion. Therefore, choosing the right parameters is crucial for studying the behavior of different states of the system.

3.2. Hidden Attractor Coexistence. In this occasion, the interesting feature, the virtual equilibrium point, will be analyzed. As we all know that nonlinear dynamical systems basically have a variety of attractive characteristic behaviors, and so does system (4). Under the same parameter variable value, different initial values can lead the trajectory to different dynamic behaviors. For some initial values, the phase trajectory can be hyperchaos. For some special initial values, the phase trajectory can converge to a very small region, similar to the fixed point, which we call the virtual 


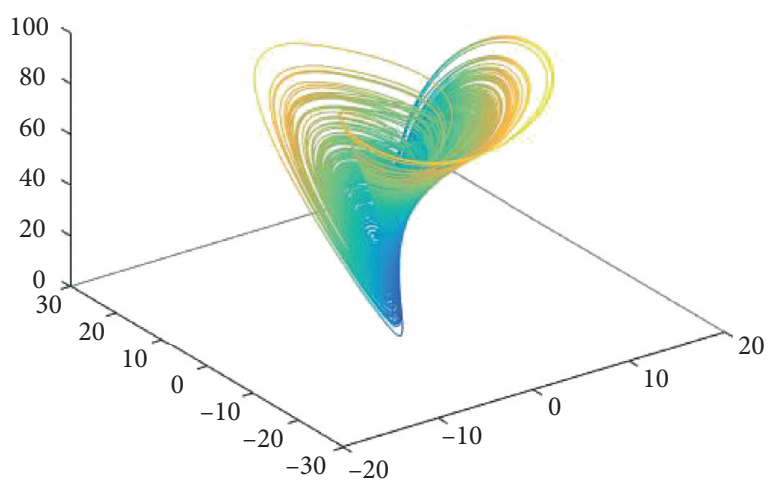

(a)

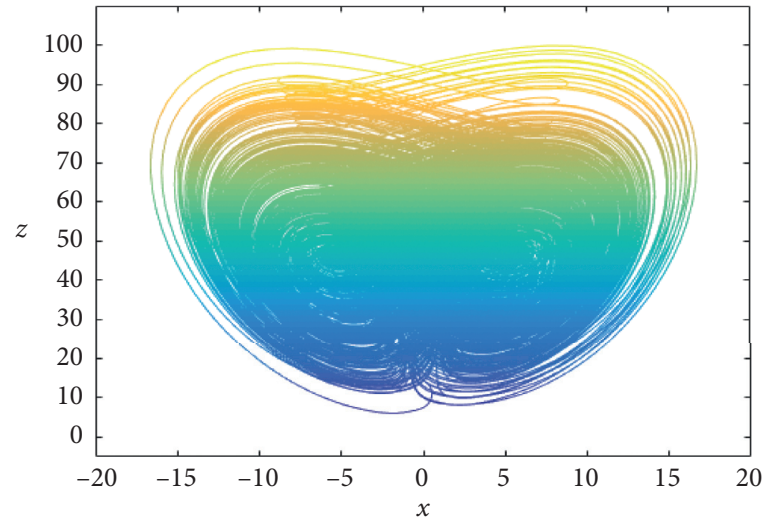

(c)

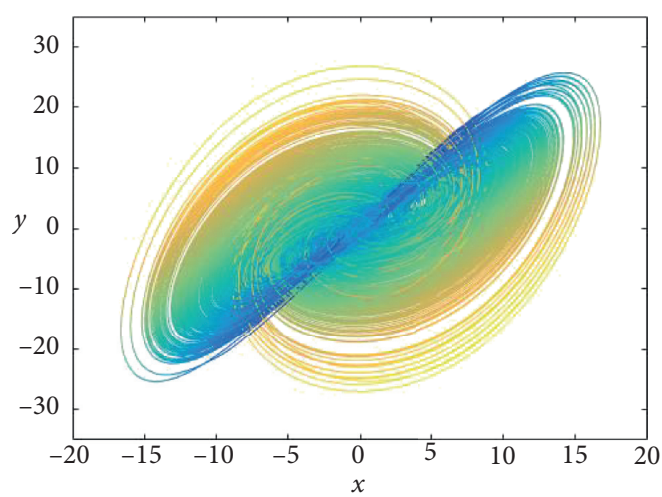

(b)

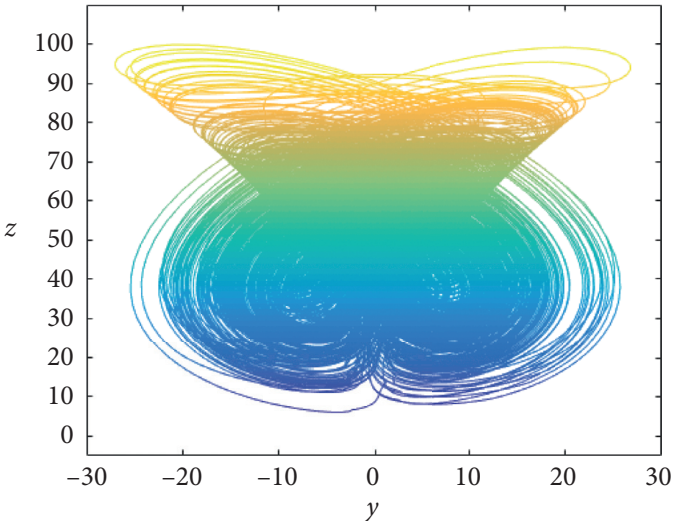

(d)

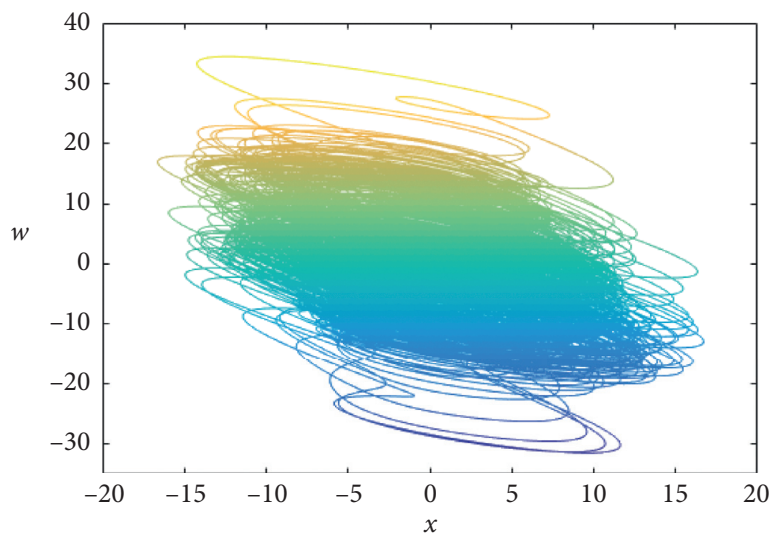

(e)

Figure 3: The chaotic system without equilibrium points for $a=10, b=40, c=2.5, k=1, h=4, g=5, d=20$, and $m=1 ; X(0)=(2.2,2.4,0.8$, 0 ): (a) in the $x-y-z$ space, (b) on the $x-y$ plane, (c) on the $x-z$ plane, (d) on the $y-z$ plane, and (e) on the $x-w$ plane.

equilibrium point. Of interesting is the fact that although it is a hidden attractor without any equilibrium points, the system still can converge to the virtual equilibrium point.

By means of numerical simulation, the time series of the trajectory starting from different initial values can be obtained, through which we can intuitively know that whether the system is chaotic or convergent. Previously analyzed, the trajectories from most initial conditions will eventually lead to a chaotic path. And only the trajectories from some initial values in a particular region will converge to the virtual equilibrium point. So two representative initial values are selected under different circumstances. With $m=1$ and $q=0.96$ and the others parameters are the same with above, the time series are shown in Figure 11, in which the blue track exhibits chaos with the initial conditions $X(0)=(2.2$, $2.4,0.8,0)$ and the track in orange was from $X(0)=(0,0,0.3$, 0.1 ) tending to the virtual equilibrium point.

Besides, the trajectories for the system are plotted in Figure 12. Under the same parameters, the system exhibits chaos behavior from $X(0)=(2.2,2.4,0.8,0)$ (in blue), while the system converges to a minimal region around $(0,0,0,0)$ corresponding to the initial conditions $X(0)=(0,0,0.3,0.1)$ 


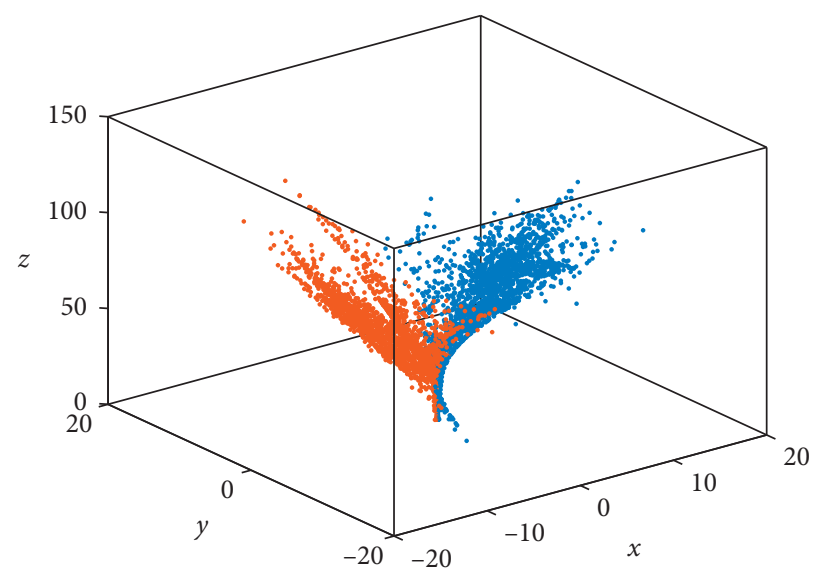

FIgURE 4: The 3D Poincare mapping of system (4).

TABLE 1: LEs of system (6) with different sampling and observation time for $a=10, b=40, c=2.5, k=1, h=4, g=5, d=20$, and $m=1$.

\begin{tabular}{lccccc}
\hline Initial condition & Observation time & Sampling time & LEs & Sum of LEs & Sign of the LEs \\
\hline $2.2,2.4,0.8,0$ & 500 & 0.02 & $1.4230,0.2312,-0.0032,-13.1211$ & -11.4611 & $(+,+,-,-)$ \\
$2.2,2.4,0.8,0$ & 2000 & 0.02 & $1.3776,0.2279,-0.0006,-13.1103$ & -11.5054 & $(+,+, 0,-)$ \\
$2.2,2.4,0.8,0$ & 5000 & 0.02 & $1.3751,0.2181,-0.0003,-13.1017$ & -11.5088 & $(+,+, 0,-)$ \\
$2.2,2.4,0.8,0$ & 500 & 0.03 & $1.4202,0.2273,-0.0072,-13.1405$ & -11.5002 & $(+,+,-,-)$ \\
$2.2,2.4,0.8,0$ & 2000 & 0.03 & $1.4135,0.2071,-0.0035,-13.1183$ & -11.5012 & $(+,+, \approx 0,-)$ \\
$2.2,2.4,0.8,0$ & 5000 & 0.03 & $1.4237,0.2065,-0.0008,-13.1156$ & -11.4862 & $(+,+, 0,-)$ \\
\hline
\end{tabular}

TABLE 2: LEs of system (4) with different sampling and observation time for $a=10, b=40, c=2.5, k=1, h=4, g=5, d=20$, and $m=20$.

\begin{tabular}{lccccc}
\hline Initial condition & Observation time & Sampling time & LEs & Sum of LEs & Sign of the LEs \\
\hline $2.2,2.4,0.8,0$ & 500 & 0.02 & $1.5721,0.0232,-0.0030,-13.5595$ & -11.9672 & $(+,+,-,-)$ \\
$2.2,2.4,0.8,0$ & 2000 & 0.02 & $1.6096,0.0098,-0.0018,-13.5789$ & -11.9613 & $(+,+, \approx 0,-)$ \\
$2.2,2.4,0.8,0$ & 5000 & 0.02 & $1.5961,0.0161,-0.0004,-13.5799$ & 11.9681 & $(+,+, 0,-)$ \\
$2.2,2.4,0.8,0$ & 500 & 0.002 & $1.6254,0.0268,-0.0028,-13.6814$ & -12.0320 & $(+,+,-,-)$ \\
$2.2,2.4,0.8,0$ & 2000 & 0.002 & $1.6354,0.0204,-0.0013,-13.6659$ & -12.0114 & $(+,+, \approx 0,-)$ \\
$2.2,2.4,0.8,0$ & 5000 & 0.002 & $1.6308,0.0145,-0.0005,-13.6501$ & 12.0053 & $(+,+, 0,-)$ \\
\hline
\end{tabular}

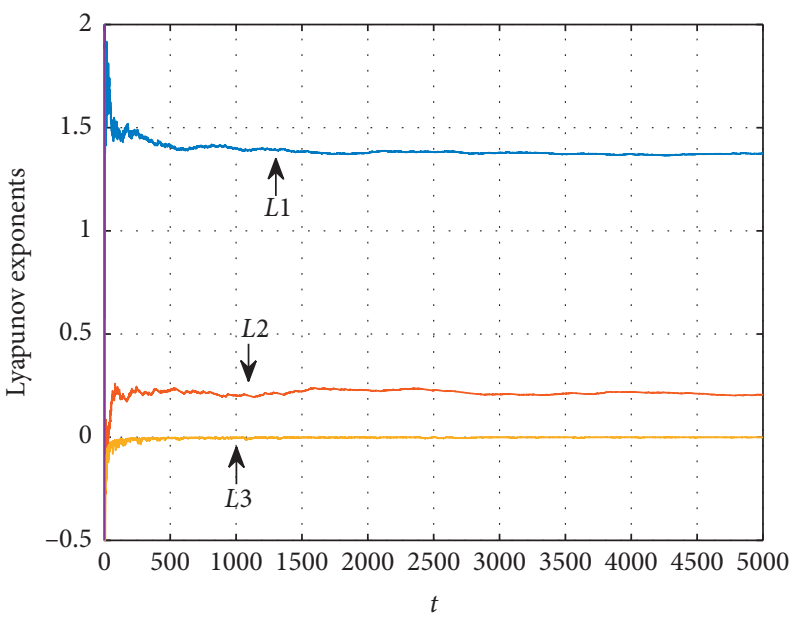

(a)

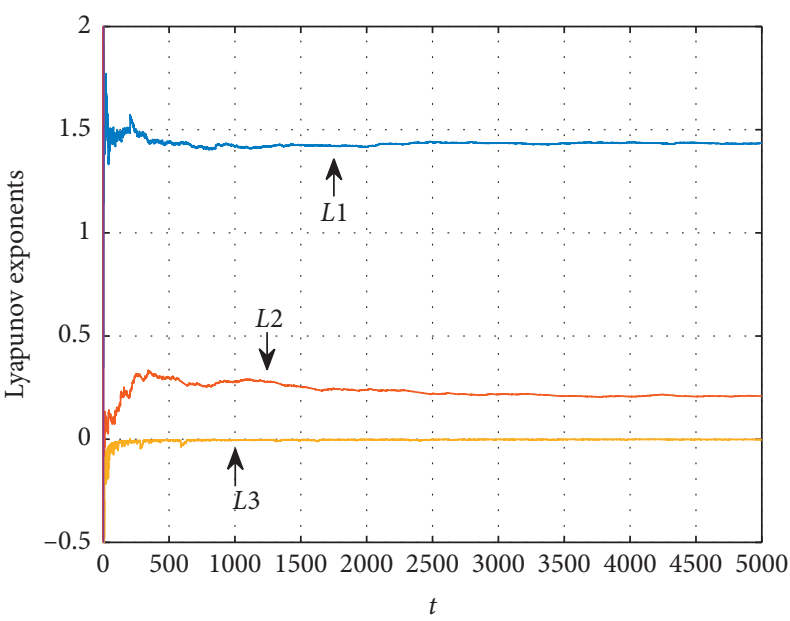

(b)

Figure 5: Lyapunov exponents of system (4) with $a=10, b=40, c=2.5, k=1, h=4, g=5, d=20$, and $m=1 ; X(0)=(2.2,2.4,0.8,0)$ : (a) with TSam $=0.02$ and (b) with TSam $=0.03$. 


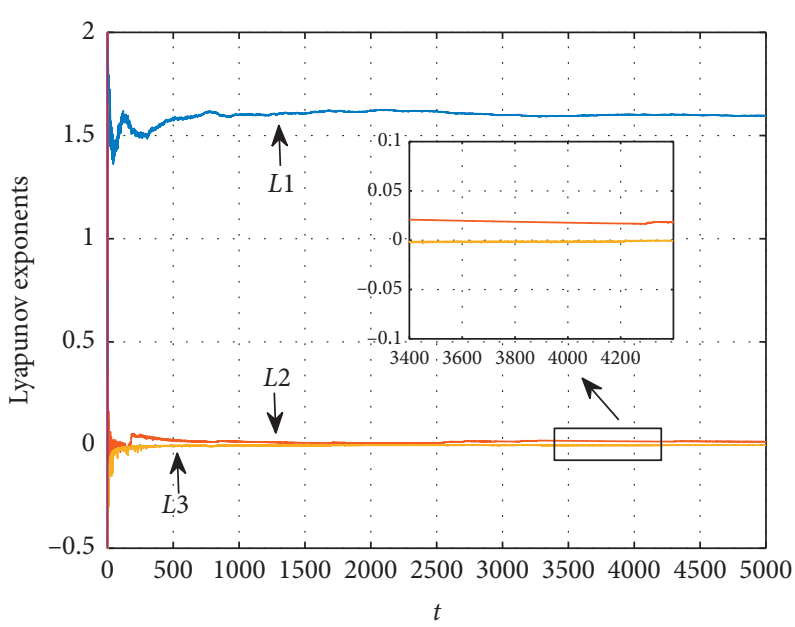

(a)

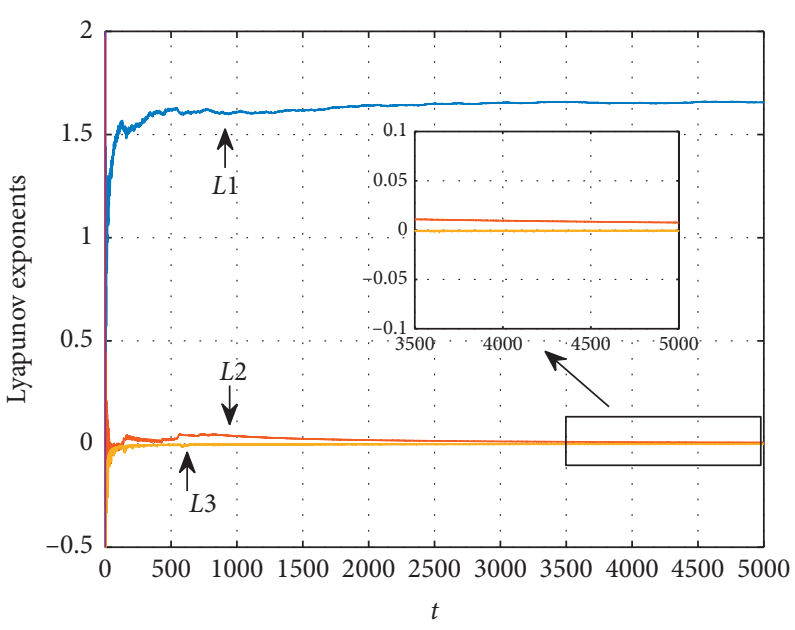

(b)

Figure 6: Lyapunov exponents of system (4) with $a=10, b=40, c=2.5, k=1, h=4, g=5, d=20$, and $m=20 ; X(0)=(2.2,2.4,0.8,0):(a)$ with $\mathrm{TSam}=0.02$ and $(\mathrm{b})$ with $\mathrm{TSam}=0.002$.

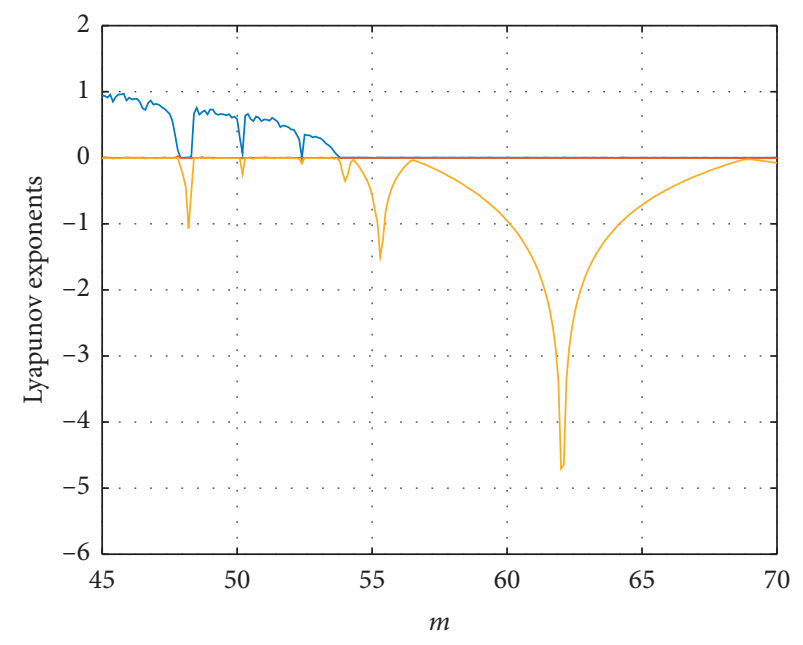

(a)

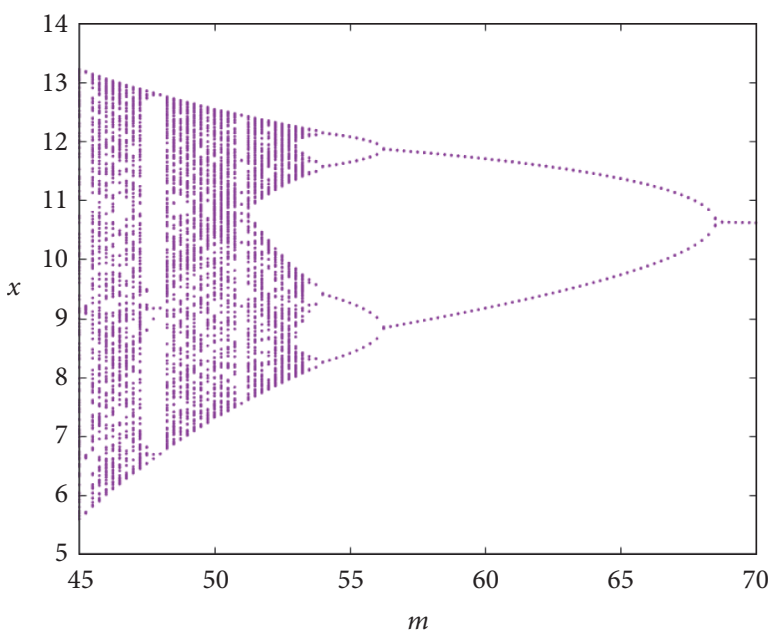

(b)

FIGURE 7: The influence of the parameter $m$ on system (4) with $a=10, b=40, c=2.5, k=1, h=4, g=5, d=20$, and $q=0.99$ : (a) the Lyapunov exponents and (b) the bifurcation.

(marked with the pentacle in orange). Figures 12(a) and 12(b) are the phase plots without transient process. And Figures 12(c) and 12(d) exhibit the transient trajectories from different initial values. It is obvious that trajectories with $(0,0,0.3,0.1)$ as initial conditions eventually converged to the minimum area near the origin which is consistent with Figure 11.

3.3. Parameter Estimation. In this section, the parameter estimations will be studied employing the new cost function for parameter estimation of chaotic models, which was proposed by Sajad Jafari in 2014 [19]. The main idea of this method is to get the regression map of the real system and the estimated system by the time series of the real system and the estimated system. Then, according to the minimum distance between the regression map of the estimation system and the regression map of the real system, the cost function is calculated to judge the estimation effect of the unknown parameters, so as to realize the estimation of the unknown parameters of the chaotic system. The new cost function can effectively overcome the sensitivity of initial conditions of the chaotic system in time domain, so it can complete the estimation of parameters under the condition that the initial conditions are not very accurate. 


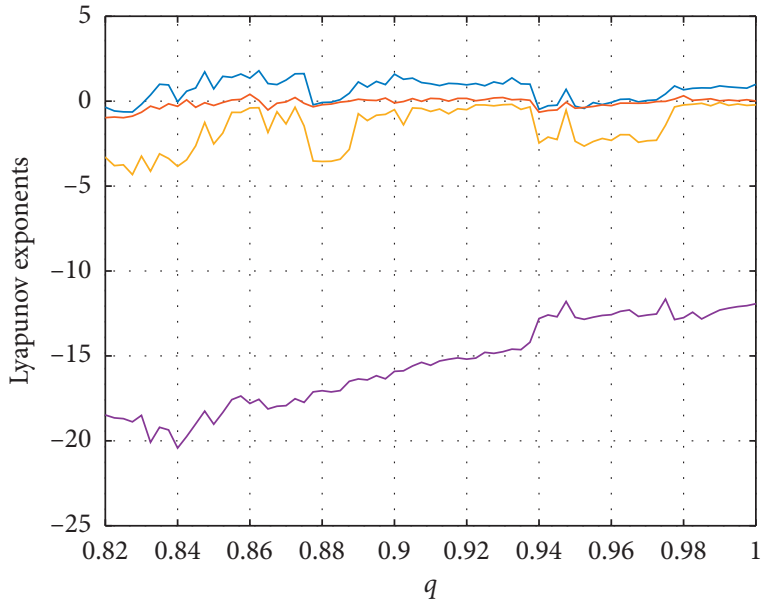

(a)

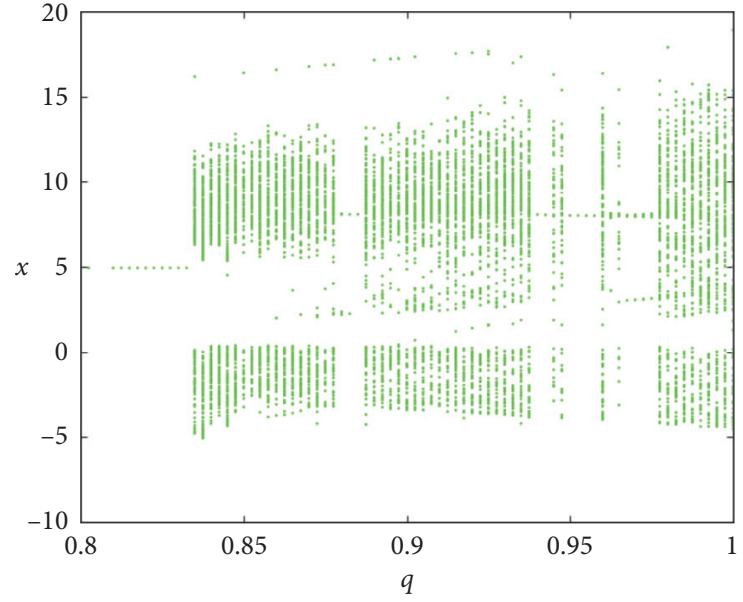

(b)

Figure 8: The influence of parameter $q$ on system (4) with $a=10, b=40, c=2.5, k=1, h=4, g=5, d=20$, and $m=15$ : (a) the Lyapunov exponents and (b) the bifurcation.

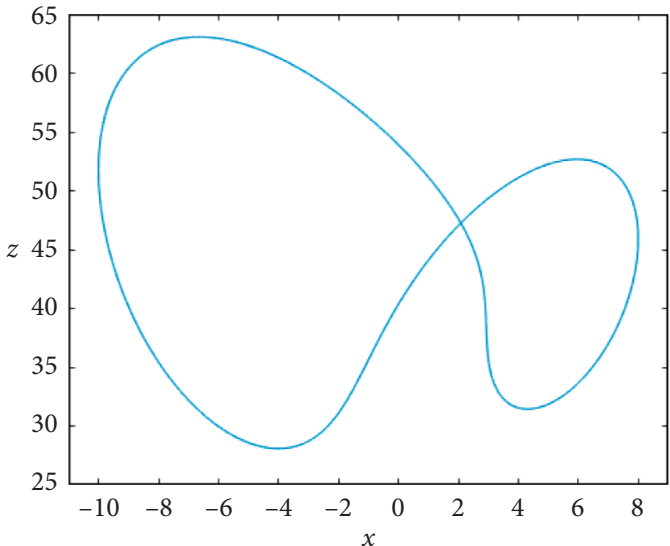

(a)

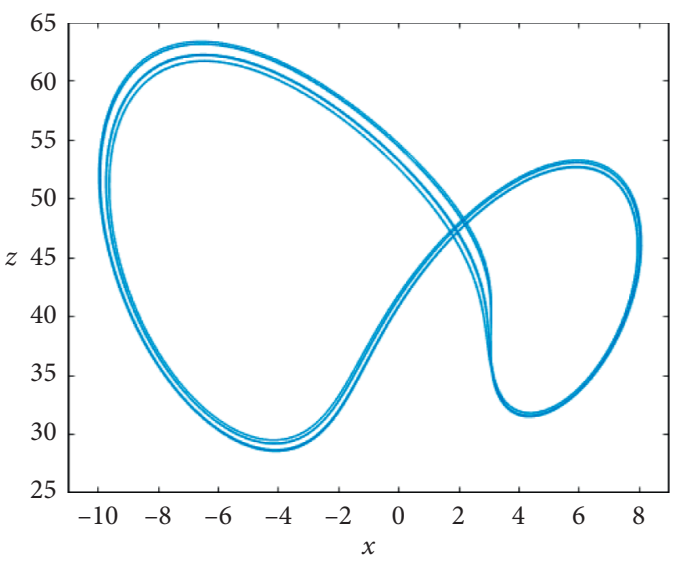

(c)

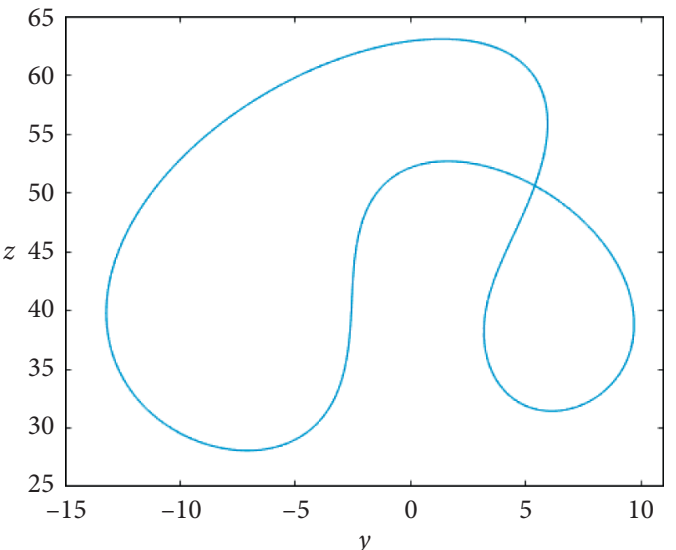

(b)

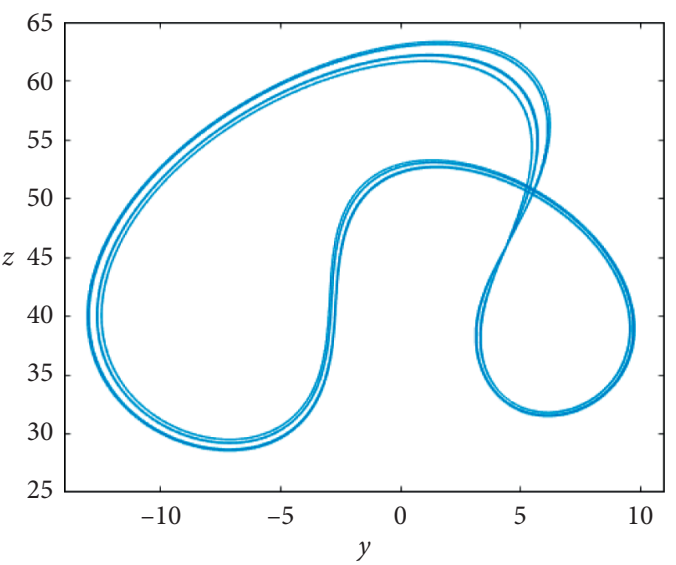

(d)

Figure 9: Continued. 


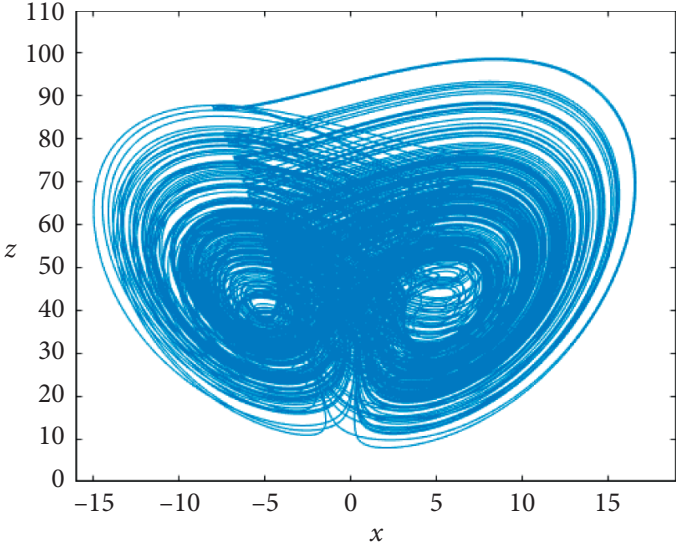

(e)

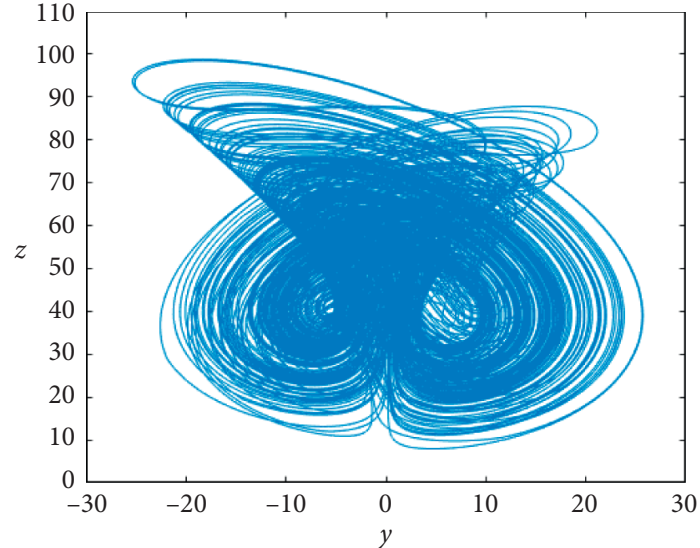

(f)

FIGURE 9: Phase plane plot of system (4) with $a=10, b=40, c=2.5, k=1, h=4, g=5, d=20$, and $m=15$ : (a) with the fractional-order $q=0.95$, (b) with the fractional-order $q=0.97$, and (c) with the fractional-order $q=0.98$.

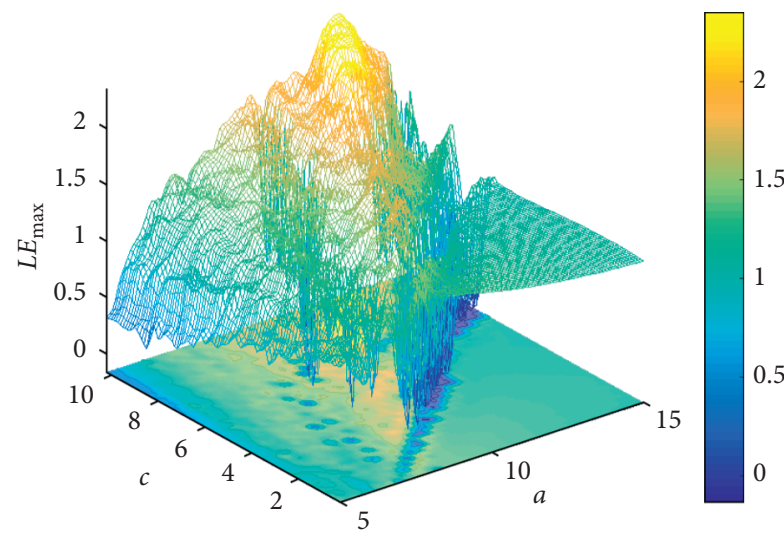

(a)

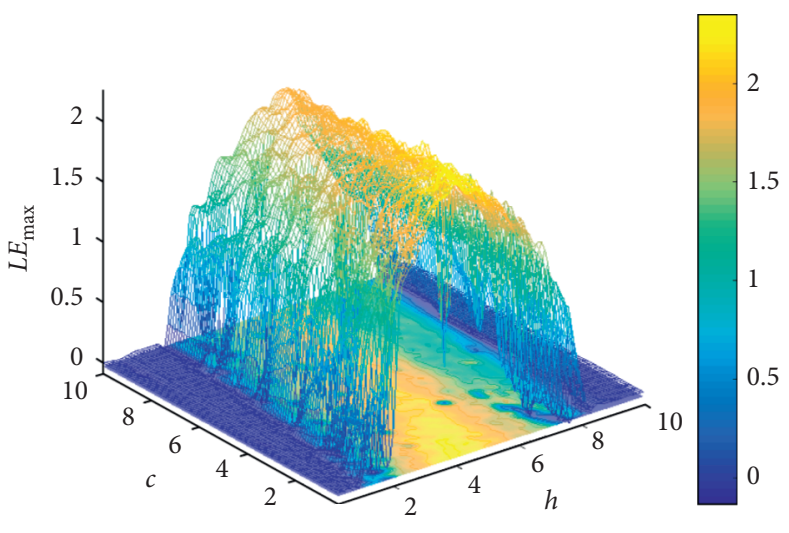

(b)

Figure 10: The largest Lyapunov exponent for system (4) with $b=40, k=1, g=5, d=20$, and $m=1$ : (a) the $a$ - $c$ section for $h=4$ and (b) the $c$ $h$ section for $a=10$.

System (4) is the actual system to be estimated, with the parameters $a=10, b=40, c=2.5, g=5, h=4, d=20$, and $m=16$ and initial conditions $(2.2,2.4,0.8,0)$. We make parameters $c$ and $h$ both unknown for estimate, and the simulation result of the cost function with parameters $c$ and $h$ is obtained as shown in Figure 13.

When the value of the cost function is the minimum, the corresponding parameter value is the estimate (where the white square is located). As can be seen in Figure 13(b), the parameter estimation results are very accurate. Different colors can generally reflect the value of the cost function and dynamical features of the system. Commonly, the parameter values of cold color region corresponding to a low cost function are unlikely to cause chaos oscillation of the system, while the parameter values of warm color region corresponding to a high cost function are more likely to cause the display of chaos of the system.

\section{Circuit Implementation of the Fractional- Order System}

Circuit designs have been used to confirm the realization ability of mathematical models [20-24]. Based on PSIM software, a circuit realization scheme of the commensurate fractional chaotic system with order $q=0.99$ and parameters $a=10, b=40, c=2.5, k=1, h=4, g=5, d=20$, and $m=1$ is designed and implemented.

The realization of the fractional-order integrator is the most important for the circuit of the fractional chaotic system. With its zeros $z_{i}$ and poles $p_{i}$, the transfer function $1 /$ sq is transformed into its zero-pole-gain form as shown in equation (7). With the transfer function approximation method proposed in [25] and $\omega_{\max }=100, P_{T}=0.01$ assumed, the zeros $z_{\mathrm{i}}$ and poles $p_{\mathrm{i}}$ are calculated as equation (8) and the expression of the fractional integrator is obtained as equation (9) under the approximate error of $\varepsilon=0.3 \mathrm{~dB}$ : 


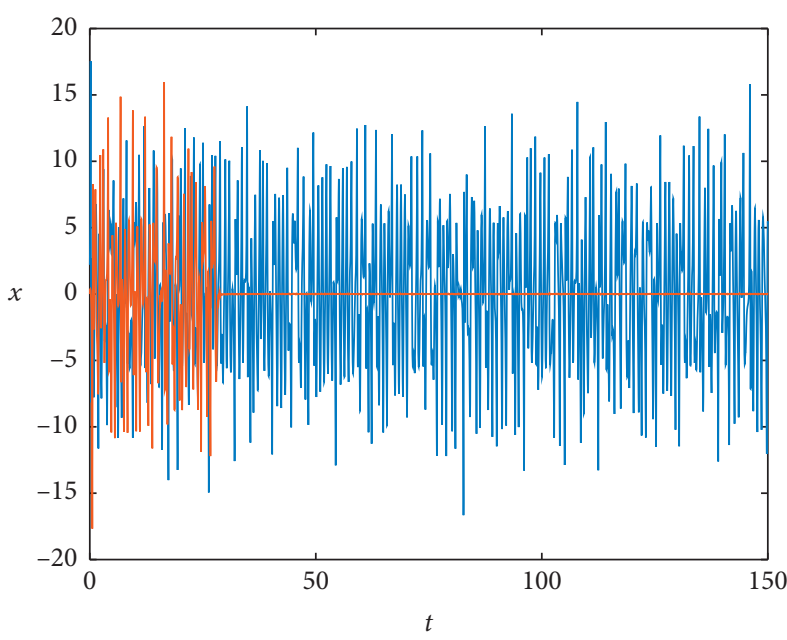

(a)

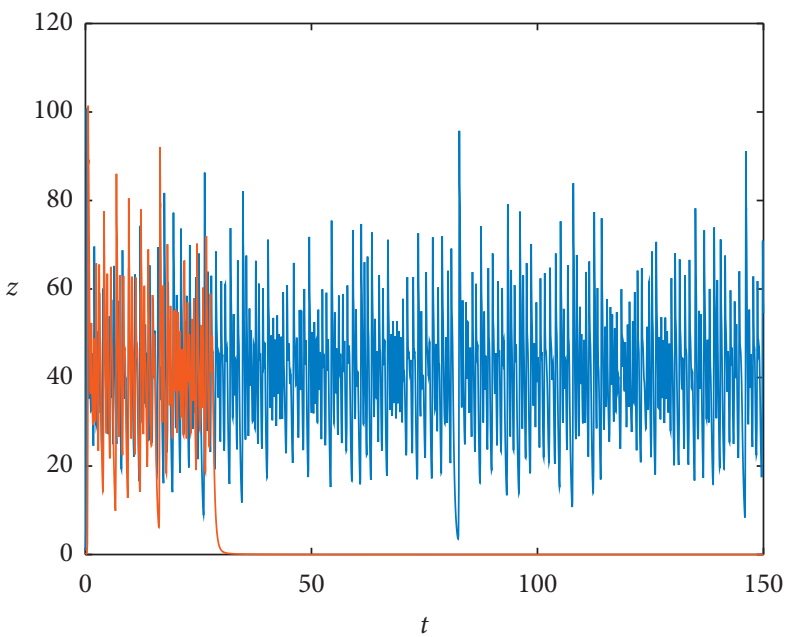

(c)

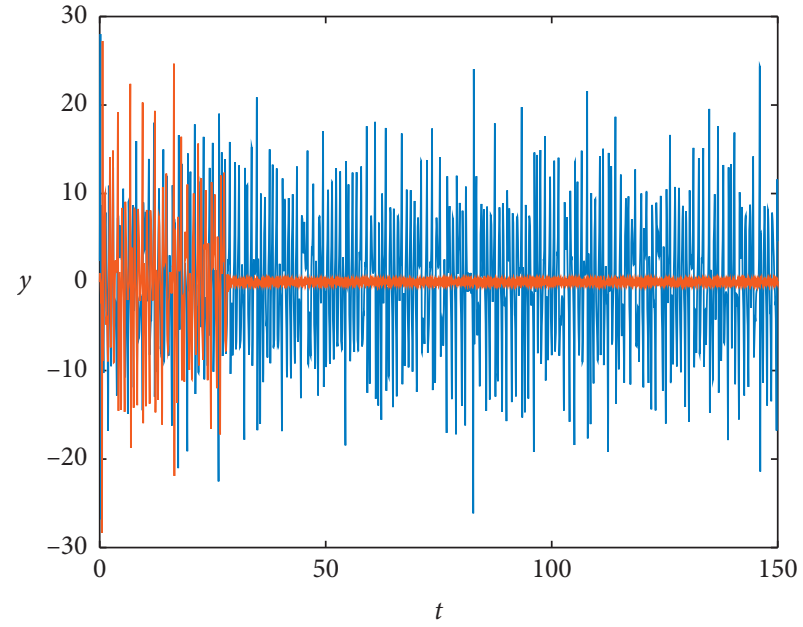

(b)

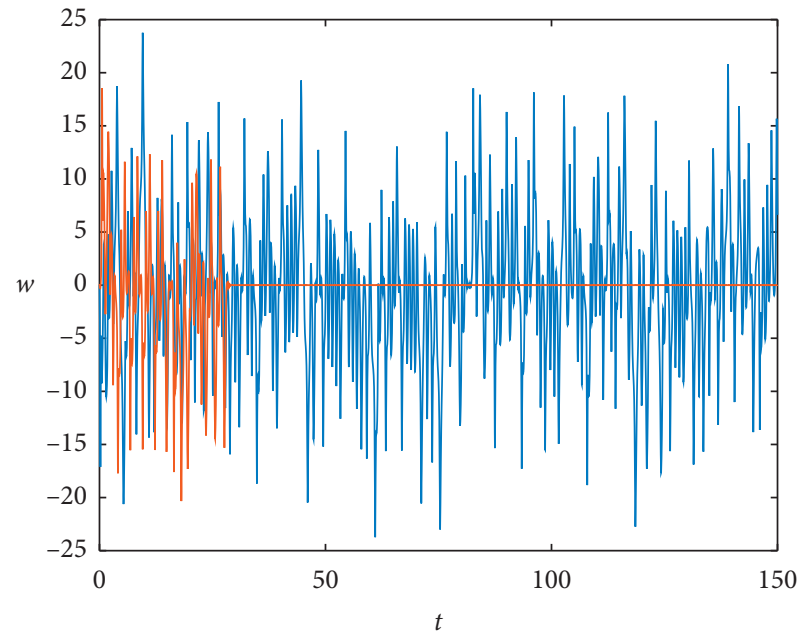

(d)

FIGURE 11: Time series of the state variables of system (4) with $a=10, b=40, c=2.5, k=1, h=4, g=5, d=20$, and $m=1$; the blue trajectories with $X(0)=(2.2,2.4,0.8,0)$ and the orange trajectories converge to zero with $X(0)=(0,0,0.3,0.1)$ : (a) the state $x$, (b) the state $y$, (c) the state $z$, and (d) the state $w$.

$F(s)=\frac{1}{\left(1+\left(s / p_{T}\right)\right)^{q}}=\frac{\prod_{i=0}^{N-1}\left(1+\left(s / z_{i}\right)\right)}{\prod_{i=0}^{N}\left(1+\left(s / p_{i}\right)\right)}$

The first pole, $\quad p_{0}=p_{T} 10^{[\varepsilon / 20 q]}$,

The first zero, $z_{0}=p_{0} 10^{[\varepsilon / 10(1-q)]}$,

The second pole, $p_{1}=z_{0} 10^{[\varepsilon / 10 q]}$,

The second zero, $z_{1}=p_{1} 10^{[\varepsilon / 10(1-q)]}$,

The third pole, $p_{2}=z_{1} 10^{[\varepsilon / 10 q]}$.

$$
\frac{1}{s^{0.99}}=\frac{1.1370(s+10.3550)(s+11103.4)}{(s+0.0104)(s+11.1034)(s+11906)} .
$$

It can be realized by three chain fractals composed of three resistor-capacitor pairs as shown in Figure 14, and its transfer function is derived as equation (10). Comparing equations (9) and (10), these parameters of electronic components are calculated as follows: $\mathrm{Ca}=1.011 \mu \mathrm{F}$, $\mathrm{Cb}=13.982 \mu \mathrm{F}, \quad \mathrm{Cc}=13.050 \mu \mathrm{F}, \quad \mathrm{Ra}=95081.958 \mathrm{k} \Omega$, $\mathrm{Rb}=6.441 \mathrm{k} \Omega$, and $\mathrm{Rc}=6.436 \Omega$ : 


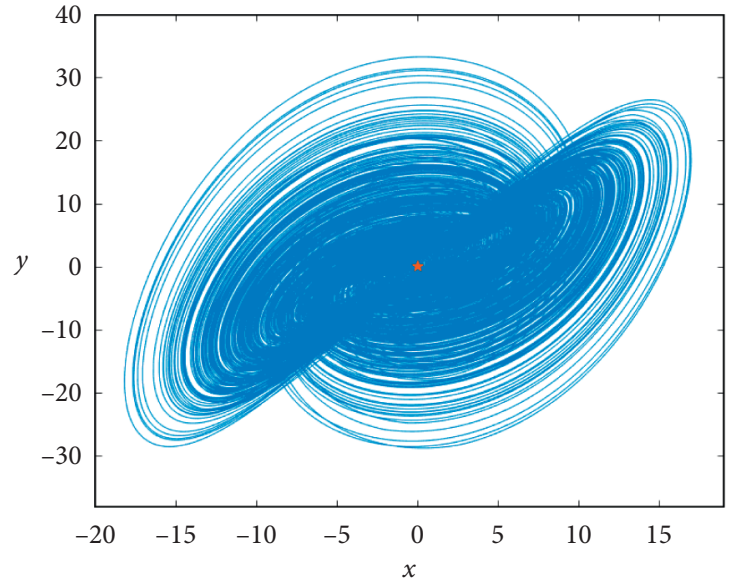

(a)

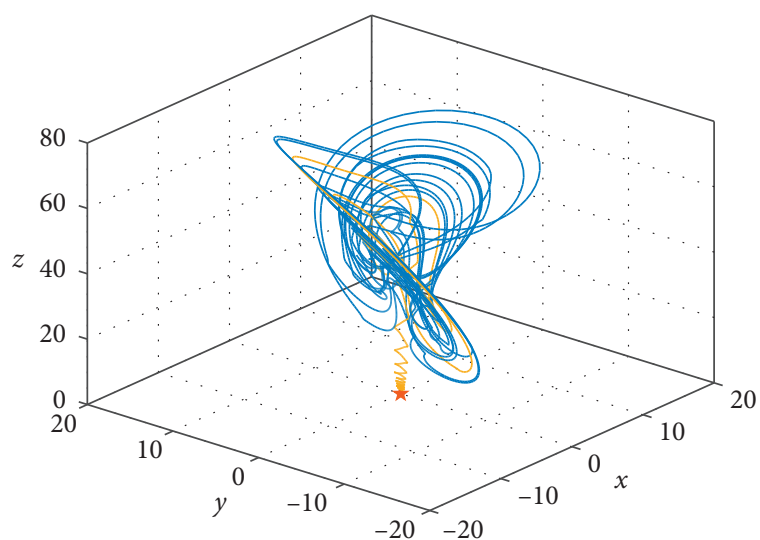

(c)

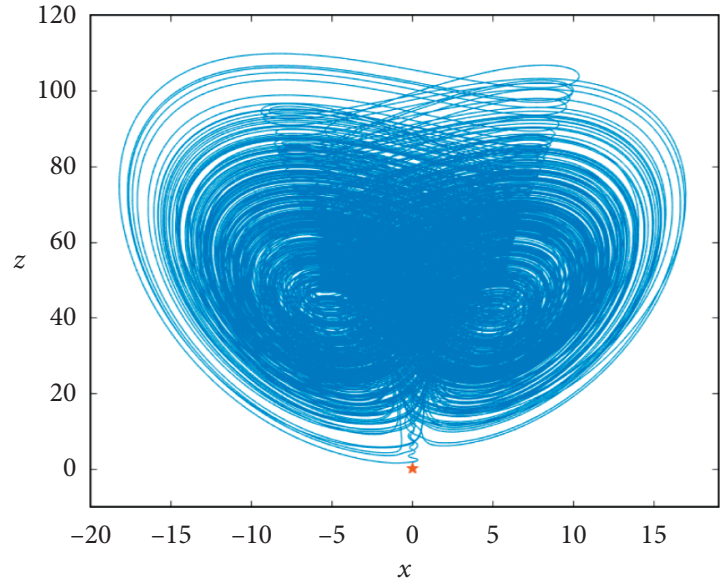

(b)

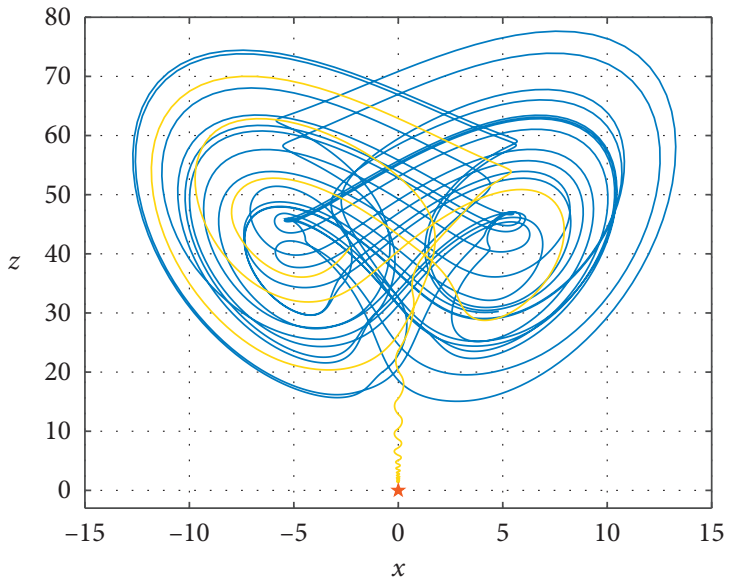

(d)

Figure 12: Coexisting attractors of system (4) with $a=10, b=40, c=2.5, k=1, h=4, g=5, d=20$, and $m=1$; the blue trajectories with $X$ $(0)=(2.2,2.4,0.8,0)$ and the yellow trajectories converge to the orange pentacle with $X(0)=(0,0,0.3,0.1)$ : (a) coexisting attractors on the $x$ $y$ plane, (b) coexisting attractors on the $x-z$ plane, (c) trajectories of the coexisting attractors in the $x-y-z$ space, and (d) trajectories of the coexisting attractors on the $\mathrm{x}-\mathrm{z}$ plane.

$$
\begin{aligned}
H(s) & =\frac{1 / C_{a}}{s+1 / R_{a} C_{a}}+\frac{1 / C_{b}}{s+1 / R_{b} C_{b}}+\frac{1 / C_{c}}{s+1 / R_{c} C_{c}} \\
& =\frac{1}{C_{0}} \frac{\left(\left(C_{0} / C_{a}\right)+\left(C_{0} / C_{b}\right)+\left(C_{0} / C_{c}\right)\right)\left[s^{2}+\left(\left(\left(C_{b}+C_{c}\right) / R_{a}\right)+\left(\left(C_{a}+C_{c}\right) / R_{b}\right)+\left(\left(C_{a}+C_{b}\right) / R_{c}\right) / C_{a} C_{b}+C_{b} C_{c}+C_{a} C_{c}\right) s+\left(\left(\left(R_{a}+R_{b}+R_{c}\right) /\left(R_{a} R_{b} R_{c}\right)\right) / C_{a} C_{b}+C_{b} C_{c}+C_{a} C_{c}\right)\right]}{\left(s+1 / R_{a} C_{a}\right)\left(s+1 / R_{b} C_{b}\right)\left(s+1 / R_{c} C_{c}\right)} .
\end{aligned}
$$

Based on the operational-amplifier approach [26], the circuit of the commensurate fractional chaotic system is shown in Figure 15. To make sure to observe the hidden attractor completely, we reduce the output of chaotic signal to $5 \%$ of the original and set

$$
\begin{aligned}
x^{\prime} & =20 x, \\
y^{\prime} & =20 y, \\
z^{\prime} & =20 z, \\
w^{\prime} & =20 w .
\end{aligned}
$$

Hence, we have $20 k=20, d / 20=1, m / 20=0.8$, and $20 h=80$. With Kirchhoff's circuit laws, the circuital equations corresponding to system (4) are described as follows: 


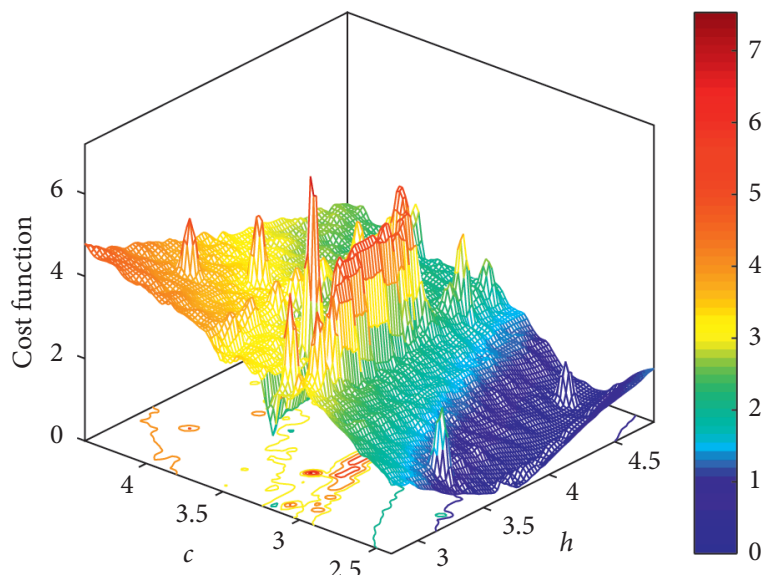

(a)

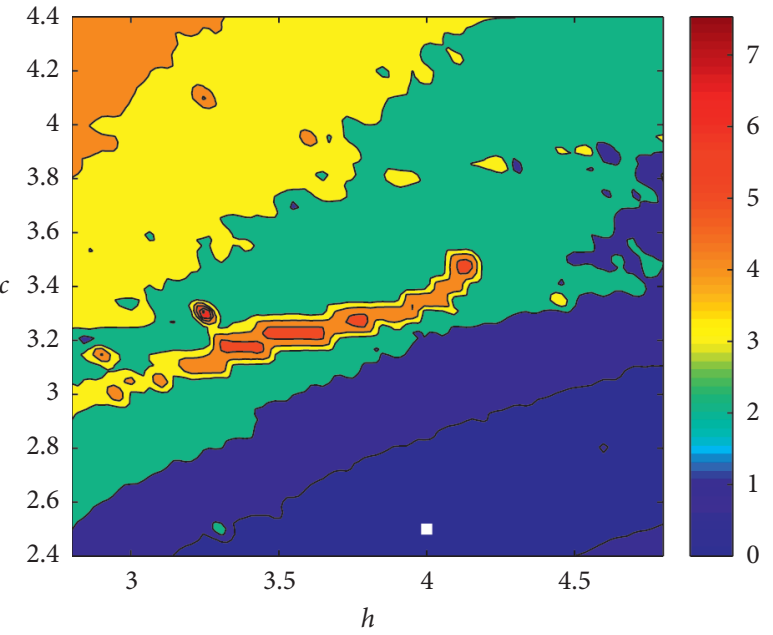

(b)

Figure 13: The cost function for system (4): (a) the $c-h$ section with $a=10, b=40, c=2.5, g=5, h=4, d=20$, and $m=16$ and (b) the contour plot of the $c-h$ section. The white square is the global minimum of the cost function.

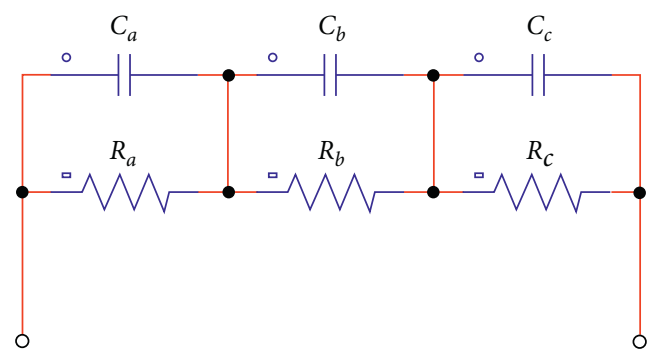

Figure 14: The electronic circuit schematic of the fractional integrator.

$$
\left\{\begin{array}{l}
\frac{\mathrm{d}^{q} x \prime}{\mathrm{d} t^{q}}=\frac{R_{3}}{C_{0} R_{1} R_{4}} y^{\prime}-\frac{R_{3} R_{6}}{C_{0} R_{2} R_{4} R_{5}} x \prime \\
\frac{\mathrm{d}^{q} y^{\prime}}{\mathrm{d} t^{q}}=\frac{R_{9}}{C_{0} R_{7} R_{10}} x \prime-\frac{R_{6} R_{9}}{C_{0} R_{5} R_{8} R_{10}} x \prime z \prime+\frac{R_{9}}{C_{0} R_{10} R_{18}} \operatorname{sign}(w \prime)+\frac{R_{9}}{C_{0} R_{10} R_{19}} V_{\mathrm{DC}}, \\
\frac{\mathrm{d}^{q} z \prime}{\mathrm{d} t^{q}}=-\frac{R_{12} R_{15}}{C_{0} R_{13} R_{14} R_{16}} z \prime+\frac{R_{6}^{2} R_{12}}{C_{0} R_{5}^{2} R_{11} R_{13}} x \prime^{2}, \\
\frac{\mathrm{d}^{q} w \prime}{\mathrm{d} t^{q}}=-\frac{1}{C_{0} R_{17}} y \prime,
\end{array}\right.
$$

where $C_{0}=1 \mu \mathrm{F}$.

The parameters of electronic components are calculated as follows: $R 1=R 3=R 5=R 7=R 12=R 14=R 15=10 \mathrm{k} \Omega$, $R 2=R 6=20 \mathrm{k} \Omega, \quad R 4=R 10=R 13=100 \mathrm{k} \Omega, \quad R 8=R 9=R 16=$ $40 \mathrm{k} \Omega, \quad R 11=5 \mathrm{k} \Omega, \quad R 17=200 \mathrm{k} \Omega, \quad R 18=400 \mathrm{k} \Omega, \quad$ and $R 19=500 \mathrm{k} \Omega$. And Figure 14 demonstrates the circuit implementation results.
Comparing with the numerical simulations in Figure 3, the hidden attractors observed in Figure 16 shrunk by around $95 \%$. Intuitively, the axis range of $z$ in Figure 16 is (0, 6 ), which is $5 \%$ of the axis range of $z$ in Figure $3(0,120)$. And the axis ranges of $x, y$, and $w$ changed in the same proportion, indicating that the circuital results are comparatively consistent with the numerical simulations. 


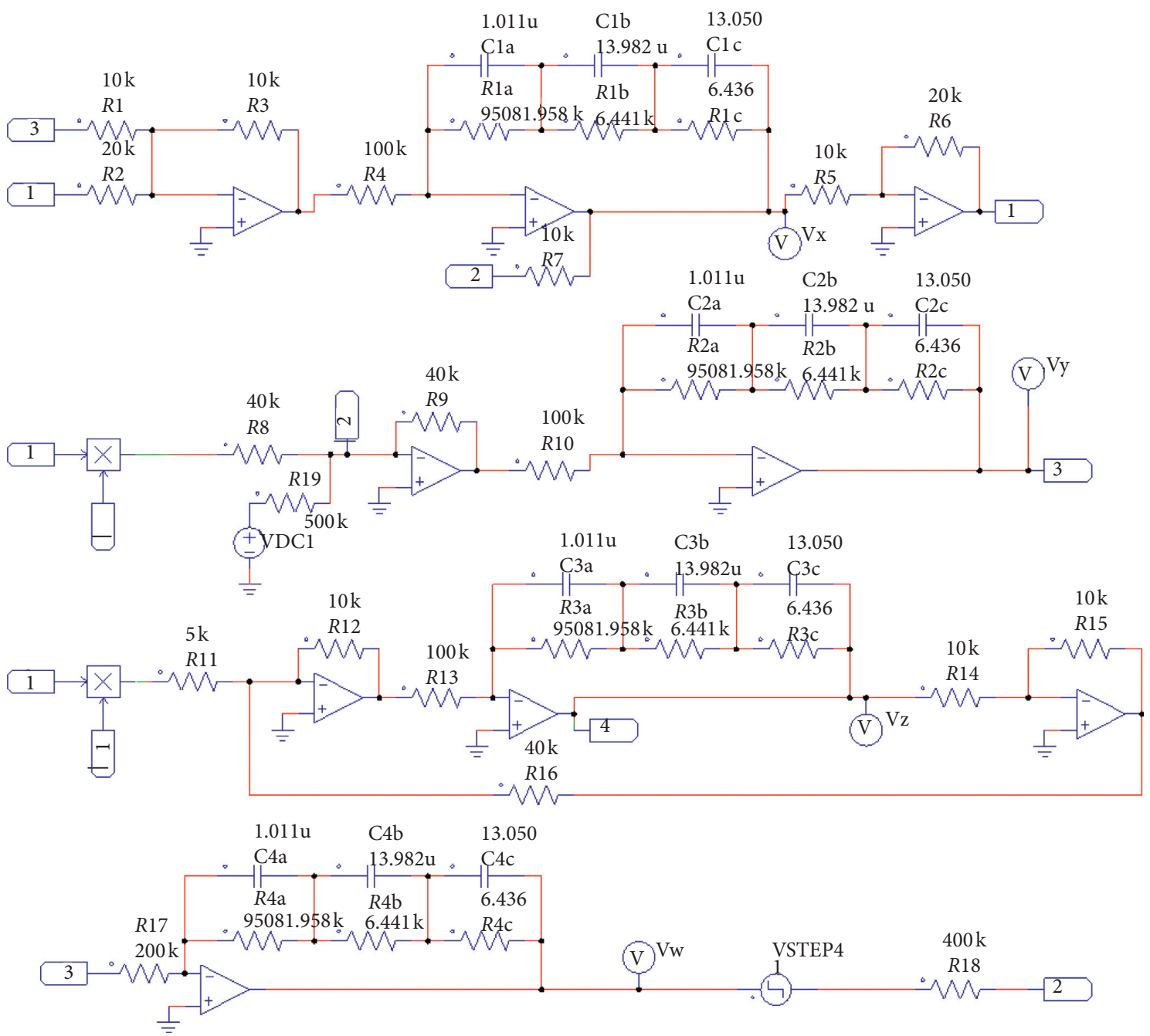

FIgUre 15: The electronic circuit schematic of fractional-order system (4) in PSIM.

\section{DSP Implementation of the Fractional- Order System}

For fractional-order chaotic systems, the design of the fractional-order integrator is crucial to its realization on the DSP platform. At present, the Adomian decomposition method (ADM for short) which is proposed in [27] is most frequently used to solve the fractional-order chaotic system $[28,29]$.

Firstly, we separate the fractional-order system into two parts:

$$
\left\{\begin{array}{c}
{ }^{*} D_{t_{0}}^{q} x(t)=L x(t)+N x(t)+g(t), \\
x^{(k)}\left(t_{0}^{+}\right)=b_{k}, \quad k=0, \ldots, m-1,
\end{array}\right.
$$

where $m=$ ceil $(q), b_{k}$ is a specified constant relating to the initial values, $\mathrm{Lx}(t)$ and $\mathrm{Nx}(t)$ are the linear and nonlinear terms of the fractional differential equations, respectively, and $g$ is the constant of the system. And the nonlinear terms can be decomposed by

$$
\left\{\begin{array}{l}
A^{i}\left(x^{0}\left(t_{n}\right), x^{1}\left(t_{n}\right), \ldots, x^{i}\left(t_{n}\right)\right)=\frac{1}{i !}\left[\frac{d^{i}}{d \lambda^{i}} N\left(v^{i}(\lambda)\right)\right]_{\lambda=0}, \\
v^{i}(\lambda)=\sum_{k=0}^{i}(\lambda)^{k} x^{k}\left(t_{n}\right),
\end{array}\right.
$$

where $i=0,1, \ldots, \infty$. Then, the nonlinear terms are expressed as

$$
N x\left(t_{n}\right)=\sum_{i=0}^{\infty} A^{i}\left(x^{0}\left(t_{n}\right), x^{1}\left(t_{n}\right), \ldots, x^{i}\left(t_{n}\right)\right) .
$$

Thus, the solution of equation (13) can be described as 


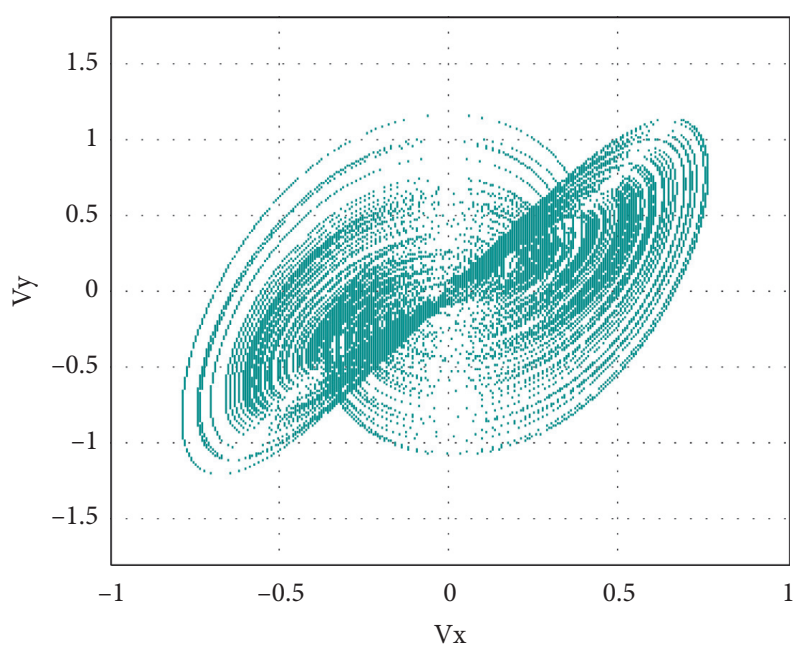

(a)

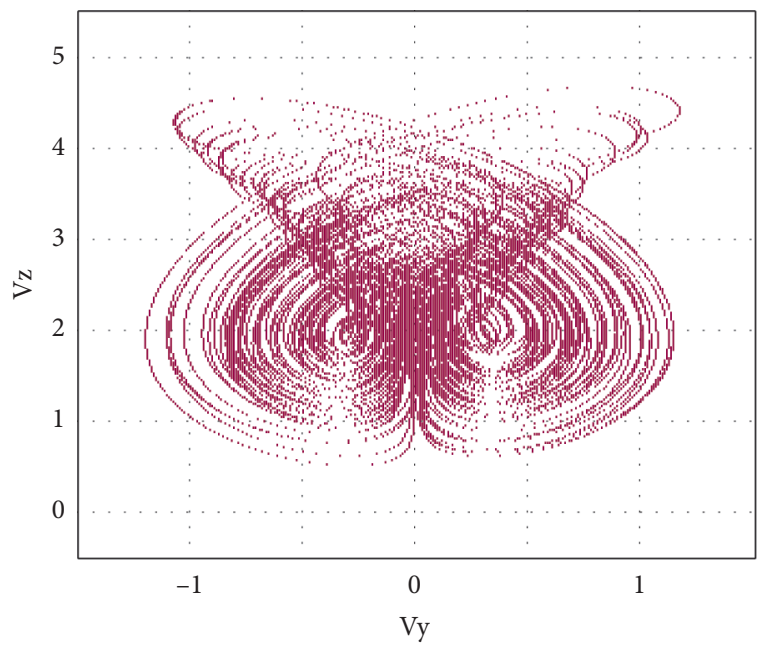

(c)

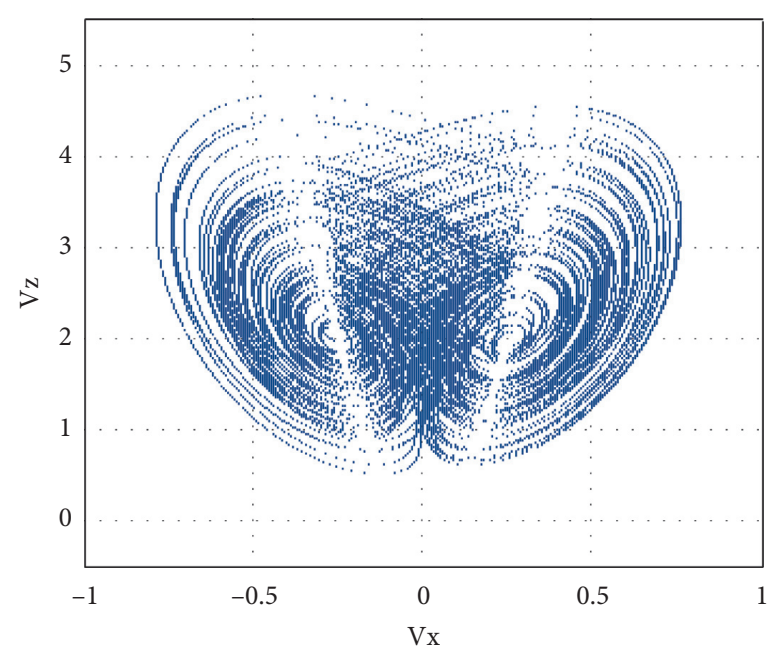

(b)

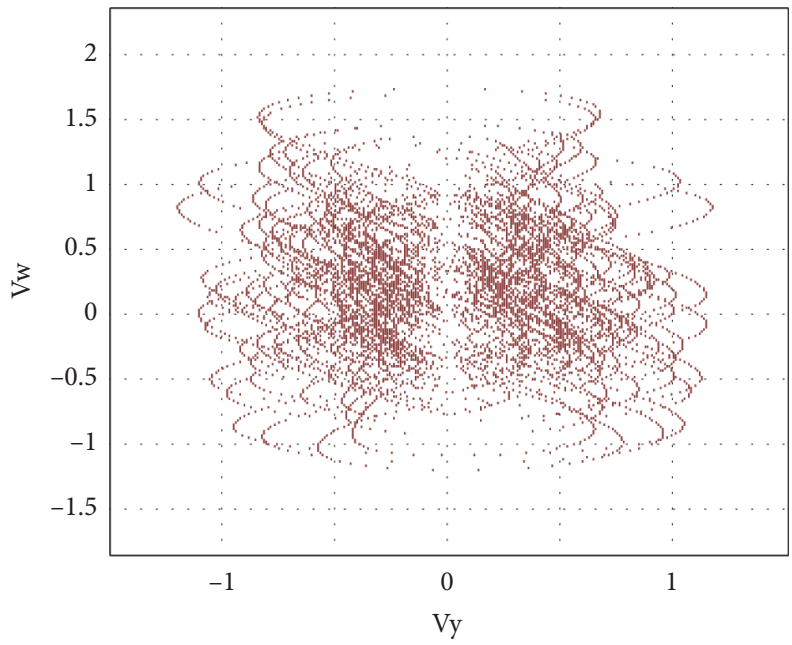

(d)

FIGURE 16: PSIM outputs of new chaotic system (4) with order $q=0.99$ and parameters $a=10, b=40, c=2.5, k=1, h=4, g=5, d=20$, and $m=1$ : (a) on the $x$-y plane, (b) on the $x-z$ plane, (c) on the $y-z$ plane, and (d) on the $x$-w plane.

$$
x\left(t_{n+1}\right)=\sum_{i=0}^{\infty} x^{i}\left(t_{n}\right)=J_{t_{0}}^{q} L \sum_{i=1}^{\infty} x^{i}\left(t_{n}\right)+J_{t_{0}}^{q} \sum_{i=1}^{\infty} A^{i}\left(t_{n}\right)+J_{t_{0}}^{q} g+\sum_{k=0}^{m-1} b_{k} \frac{\left(t-t_{0}\right)^{k}}{k !} .
$$

The method to get each decomposition item is derived by

$$
\left\{\begin{array}{l}
x^{0}\left(t_{n}\right)=x\left(t_{n}\right)+J_{t_{0}}^{q} g, \\
x^{1}\left(t_{n}\right)=J_{t_{n}}^{q} L x^{0}\left(t_{n}\right)+J_{t_{n}}^{q} \mathbf{A}^{0}\left(x^{0}\left(t_{n}\right)\right), \\
x^{2}\left(t_{n}\right)=J_{t_{n}}^{q} L x^{1}\left(t_{n}\right)+J_{t_{n}}^{q} \mathbf{A}^{1}\left(x^{0}\left(t_{n}\right), x^{1}\left(t_{n}\right)\right), \\
\vdots \\
x^{i}\left(t_{n}\right)=J_{t_{n}}^{q} L x^{i-1}\left(t_{n}\right)+J_{t_{n}}^{q} \mathbf{A}^{i-1}\left(x^{0}\left(t_{n}\right), x^{1}\left(t_{n}\right), \cdots, x^{i-1}\left(t_{n}\right)\right) . \\
\vdots
\end{array}\right.
$$

According to equations (13)-(17), the discrete solution of the fractional-order chaotic system can be denoted as $\mathbf{x}$ $(n+1)=F(\mathbf{x}(n))$. Because of its high accuracy, fast convergence, and less computer resource consumption, the
ADM becomes one of the best candidate methods to decompose the nonlinear differential equations [30].

The fractional-order chaotic system is

$$
\left\{\begin{array}{l}
{ }^{*} D_{t_{0}}^{q} x=10(y-x), \\
{ }^{*} D_{t_{0}}^{q} y=40 x-x z+20 \operatorname{sign}(w)+m, \\
{ }^{*} D_{t_{0}}^{q} z=-2.5 z+4 x^{2}, \\
{ }^{*} D_{t_{0}}^{q} w=-5 y .
\end{array}\right.
$$

By employing the ADM and setting step size $h=t_{n+1}-\mathrm{t}_{n}$, the discrete iterative formulas of proposed system (18) can be obtained. Considering the fast convergence performance of the ADM, the first six terms of the iteration are selected: 


$$
\left[\begin{array}{l}
x\left(t_{n+1}\right) \\
y\left(t_{n+1}\right) \\
z\left(t_{n+1}\right) \\
w\left(t_{n+1}\right)
\end{array}\right]=\left[\begin{array}{c}
x^{0}\left(t_{n}\right)+x^{1}\left(t_{n}\right)+x^{2}\left(t_{n}\right)+x^{3}\left(t_{n}\right)+x^{4}\left(t_{n}\right)+x^{5}\left(t_{n}\right) \\
y^{0}\left(t_{n}\right)+y^{1}\left(t_{n}\right)+y^{2}\left(t_{n}\right)+y^{3}\left(t_{n}\right)+y^{4}\left(t_{n}\right)+y^{5}\left(t_{n}\right) \\
z^{0}\left(t_{n}\right)+z^{1}\left(t_{n}\right)+z^{2}\left(t_{n}\right)+z^{3}\left(t_{n}\right)+z^{4}\left(t_{n}\right)+z^{5}\left(t_{n}\right) \\
w^{0}\left(t_{n}\right)+w^{1}\left(t_{n}\right)+w^{2}\left(t_{n}\right)+w^{3}\left(t_{n}\right)+w^{4}\left(t_{n}\right)+w^{5}\left(t_{n}\right)
\end{array}\right] .
$$

According to the given conditions, the following equation can be obtained:

$$
\begin{aligned}
& x^{0}\left(t_{n}\right)=x\left(t_{n}\right), \\
& y^{0}\left(t_{n}\right)=y\left(t_{n}\right)+m \frac{h^{q}}{\Gamma(q+1)}, \\
& z^{0}\left(t_{n}\right)=z\left(t_{n}\right), \\
& w^{0}\left(t_{n}\right)=w\left(t_{n}\right),
\end{aligned}
$$

where $\Gamma(\cdot)$ is the Gamma function.

Make $c_{1}^{0}=x^{0}\left(t_{n}\right)=x\left(t_{n}\right), c_{2}^{0}=y\left(t_{n}\right), c_{3}^{0}=z^{0}\left(t_{n}\right)=$ $z\left(t_{n}\right)$, and $c_{4}^{0}=w^{0}\left(t_{n}\right)=w\left(t_{n}\right)$, and the second term of each state variable is shown as follows:

$$
\begin{aligned}
& x^{1}\left(t_{n}\right)=c_{1}^{1} \frac{h^{q}}{\Gamma(q+1)}+c_{1}^{10} \frac{h^{2 q}}{\Gamma(2 q+1)}, \\
& y^{1}\left(t_{n}\right)=c_{2}^{1} \frac{h^{q}}{\Gamma(q+1)}, \\
& z^{1}\left(t_{n}\right)=c_{3}^{1} \frac{h^{q}}{\Gamma(q+1)}, \\
& w^{1}\left(t_{n}\right)=c_{4}^{1} \frac{h^{q}}{\Gamma(q+1)}+c_{4}^{10} \frac{h^{2 q}}{\Gamma(2 q+1)},
\end{aligned}
$$

where

$$
\begin{aligned}
c_{1}^{1} & =10\left(c_{2}^{0}-c_{1}^{0}\right), \\
c_{1}^{10} & =10 m, \\
c_{2}^{1} & =40 c_{1}^{0}-c_{1}^{0} c_{3}^{0}+20 \operatorname{sign}\left(c_{4}^{0}\right), \\
c_{3}^{1} & =-2.5 c_{3}^{0}+4\left(c_{1}^{0}\right)^{2}, \\
c_{4}^{1} & =-5 c_{2}^{0} \\
c_{4}^{10} & =-5 m .
\end{aligned}
$$

Similarly, the component $x^{2}$ is

$$
\begin{aligned}
& x^{2}\left(t_{n}\right)=c_{1}^{2} \frac{h^{2 q}}{\Gamma(2 q+1)}+c_{1}^{20} \frac{h^{3 q}}{\Gamma(3 q+1)}, \\
& y^{2}\left(t_{n}\right)=c_{2}^{2} \frac{h^{2 q}}{\Gamma(2 q+1)}+c_{2}^{20} \frac{h^{3 q}}{\Gamma(3 q+1)}, \\
& z^{2}\left(t_{n}\right)=c_{3}^{2} \frac{h^{2 q}}{\Gamma(2 q+1)}+c_{3}^{20} \frac{h^{3 q}}{\Gamma(3 q+1)}, \\
& w^{2}\left(t_{n}\right)=c_{4}^{2} \frac{h^{2 q}}{\Gamma(2 q+1)}
\end{aligned}
$$

where

$$
\begin{aligned}
c_{1}^{2} & =10\left(c_{2}^{1}-c_{1}^{1}\right), \\
c_{1}^{20} & =-10 c_{1}^{10}, \\
c_{2}^{2} & =40 c_{1}^{1}-c_{1}^{0} c_{3}^{1}-c_{1}^{1} c_{3}^{0}+20 \operatorname{sign}\left(c_{4}^{1}\right), \\
c_{2}^{20} & =40 c_{1}^{10}-c_{1}^{10} c_{3}^{0}+20 \operatorname{sign}\left(c_{4}^{10}\right), \\
c_{3}^{2} & =-2.5 c_{3}^{1}+8 c_{1}^{0} c_{1}^{1}, \\
c_{3}^{20} & =8 c_{0}^{1} c_{1}^{10}, \\
c_{4}^{2} & =-5 c_{2}^{1} .
\end{aligned}
$$

By applying the same approach to the components $x^{3}$ and $x^{4}$, the component $x^{5}$ is

$$
\begin{aligned}
& x^{3}\left(t_{n}\right)=c_{1}^{3} \frac{h^{3 q}}{\Gamma(3 q+1)}+c_{1}^{30} \frac{h^{4 q}}{\Gamma(4 q+1)}, \\
& y^{3}\left(t_{n}\right)=c_{2}^{3} \frac{h^{3 q}}{\Gamma(3 q+1)}+c_{2}^{30} \frac{h^{4 q}}{\Gamma(4 q+1)}, \\
& z^{3}\left(t_{n}\right)=c_{3}^{3} \frac{h^{3 q}}{\Gamma(3 q+1)}+c_{3}^{30} \frac{h^{4 q}}{\Gamma(4 q+1)}+c_{3}^{31} \frac{h^{5 q}}{\Gamma(5 q+1)^{\prime}}, \\
& w^{3}\left(t_{n}\right)=c_{4}^{3} \frac{h^{3 q}}{\Gamma(3 q+1)}+c_{4}^{30} \frac{h^{4 q}}{\Gamma(4 q+1)},
\end{aligned}
$$

where 


$$
\begin{aligned}
c_{1}^{3} & =10\left(c_{2}^{2}-c_{1}^{2}\right), \\
c_{1}^{20} & =10\left(c_{2}^{20}-c_{1}^{20}\right), \\
c_{2}^{3} & =40 c_{1}^{2}-c_{1}^{0} c_{3}^{2}-c_{1}^{2} c_{3}^{0}-c_{1}^{1} c_{3}^{1} \frac{\Gamma(2 q+1)}{\Gamma^{2}(q+1)}+20 \operatorname{sign}\left(c_{4}^{2}\right), \\
c_{2}^{30} & =40 c_{1}^{20}-c_{1}^{0} c_{3}^{20}-c_{1}^{20} c_{3}^{0}-c_{1}^{10} c_{3}^{1} \frac{\Gamma(3 q+1)}{\Gamma(q+1) \Gamma(2 q+1)}, \\
c_{3}^{3} & =-2.5 c_{3}^{2}+8 c_{1}^{0} c_{1}^{2}+4\left(c_{1}^{1}\right)^{2} \frac{\Gamma(2 q+1)}{\Gamma^{2}(q+1)}, \\
c_{3}^{30} & =-2.5 c_{3}^{20}+8 c_{1}^{0} c_{1}^{20}+8 c_{1}^{1} c_{1}^{10} \frac{\Gamma(3 q+1)}{\Gamma(q+1) \Gamma(2 q+1)}, \\
c_{3}^{31} & =4\left(c_{1}^{10}\right)^{2} \frac{\Gamma(4 q+1)}{\Gamma^{2}(2 q+1)}, \\
c_{4}^{3} & =-5 c_{2}^{2}, \quad c_{4}^{30} \\
x^{4}\left(t_{n}\right) & =c_{1}^{4} \frac{h^{4 q}}{\Gamma(4 q+1)}+c_{1}^{40} \frac{h^{5 q}}{\Gamma(5 q+1)}, \\
y^{4}\left(t_{n}\right) & =c_{2}^{4} \frac{h^{4 q}}{\Gamma(4 q+1)}+c_{2}^{40} \frac{h^{5 q}}{\Gamma(5 q+1)}+c_{2}^{41} \frac{h^{6 q}}{\Gamma(6 q+1)}, \\
z^{4}\left(t_{n}\right) & =c_{3}^{4} \frac{h^{4 q}}{\Gamma(4 q+1)}+c_{3}^{40} \frac{h^{5 q}}{\Gamma(5 q+1)}+c_{3}^{41} \frac{h^{6 q}}{\Gamma(6 q+1)}, \\
w^{4}\left(t_{n}\right) & =c_{4}^{4} \frac{h^{4 q}}{\Gamma(4 q+1)}+c_{4}^{40} \frac{h^{5 q}}{\Gamma(5 q+1)},
\end{aligned}
$$

where

$$
\begin{aligned}
& c_{1}^{4}=10\left(c_{2}^{3}-c_{1}^{3}\right), \\
& c_{1}^{40}=10\left(c_{2}^{30}-c_{1}^{30}\right), \\
& c_{2}^{4}=40 c_{1}^{3}-c_{1}^{0} c_{3}^{3}-c_{1}^{2} c_{3}^{0}-c_{1}^{3} c_{3}^{0}+\left(-c_{1}^{1} c_{3}^{2}-c_{1}^{2} c_{3}^{1}\right) \frac{\Gamma(3 q+1)}{\Gamma(q+1) \Gamma(2 q+1)}+20 \operatorname{sign}\left(c_{4}^{3}\right), \\
& c_{2}^{40}=40 c_{1}^{30}-c_{1}^{0} c_{3}^{30}-c_{1}^{30} c_{3}^{0}+\left(-c_{1}^{1} c_{3}^{20}-c_{1}^{20} c_{3}^{1}\right) \frac{\Gamma(4 q+1)}{\Gamma(q+1) \Gamma(3 q+1)}-c_{1}^{10} c_{3}^{2} \frac{\Gamma(4 q+1)}{\Gamma^{2}(2 q+1)}+20 \operatorname{sign}\left(c_{4}^{30}\right), \\
& c_{2}^{41}=-c_{1}^{0} c_{3}^{31}-c_{1}^{10} c_{3}^{20} \frac{\Gamma(5 q+1)}{\Gamma(2 q+1) \Gamma(3 q+1)}, \\
& c_{3}^{4}=-2.5 c_{3}^{3}+8 c_{1}^{0} c_{1}^{3}+8 c_{1}^{1} c_{1}^{2} \frac{\Gamma(3 q+1)}{\Gamma(q+1) \Gamma(2 q+1)}, \\
& c_{3}^{40}=-2.5 c_{3}^{30}+8 c_{1}^{0} c_{1}^{30}+8 c_{1}^{1} c_{1}^{20} \frac{\Gamma(4 q+1)}{\Gamma(q+1) \Gamma(3 q+1)}+8 c_{1}^{10} c_{1}^{2} \frac{\Gamma(4 q+1)}{\Gamma^{2}(2 q+1)}, \\
& c_{3}^{41}=-2.5 c_{3}^{31}+8 c_{1}^{10} c_{1}^{20} \frac{\Gamma(5 q+1)}{\Gamma(2 q+1) \Gamma(3 q+1)}, \\
& c_{4}^{4}=-5 c_{2}^{3}, \\
& c_{4}^{40}=-5 c_{2}^{30} .
\end{aligned}
$$




$$
\begin{aligned}
& x^{5}\left(t_{n}\right)=c_{1}^{5} \frac{h^{5 q}}{\Gamma(5 q+1)}+c_{1}^{50} \frac{h^{6 q}}{\Gamma(6 q+1)}+c_{1}^{51} \frac{h^{7 q}}{\Gamma(7 q+1)}, \\
& y^{5}\left(t_{n}\right)=c_{2}^{5} \frac{h^{5 q}}{\Gamma(5 q+1)}+c_{2}^{50} \frac{h^{6 q}}{\Gamma(6 q+1)}+c_{2}^{51} \frac{h^{7 q}}{\Gamma(7 q+1)}+c_{2}^{52} \frac{h^{8 q}}{\Gamma(8 q+1)}, \\
& z^{5}\left(t_{n}\right)=c_{3}^{5} \frac{h^{5 q}}{\Gamma(5 q+1)}+c_{3}^{50} \frac{h^{6 q}}{\Gamma(6 q+1)}+c_{3}^{51} \frac{h^{7 q}}{\Gamma(7 q+1)}, \\
& w^{5}\left(t_{n}\right)=c_{4}^{5} \frac{h^{5 q}}{\Gamma(5 q+1)}+c_{4}^{50} \frac{h^{6 q}}{\Gamma(6 q+1)}+c_{4}^{51} \frac{h^{7 q}}{\Gamma(7 q+1)},
\end{aligned}
$$

where

$$
\begin{aligned}
& c_{1}^{5}=10\left(c_{2}^{4}-c_{1}^{4}\right) \\
& c_{1}^{50}=10\left(c_{2}^{40}-c_{1}^{40}\right) \\
& c_{1}^{51}=10 c_{2}^{41} \\
& c_{2}^{5}=40 c_{1}^{4}-c_{1}^{0} c_{3}^{4}-c_{1}^{4} c_{3}^{0}+\left(-c_{1}^{1} c_{3}^{3}-c_{1}^{3} c_{3}^{1}\right) \frac{\Gamma(4 q+1)}{\Gamma(q+1) \Gamma(3 q+1)}-c_{1}^{2} c_{3}^{2} \frac{\Gamma(4 q+1)}{\Gamma^{2}(2 q+1)}+20 \operatorname{sign}\left(c_{4}^{4}\right), \\
& c_{2}^{50}=40 c_{1}^{40}-c_{1}^{0} c_{3}^{40}-c_{1}^{40} c_{3}^{0}+\left(-c_{1}^{1} c_{3}^{30}-c_{1}^{30} c_{3}^{1}\right) \frac{\Gamma(5 q+1)}{\Gamma(q+1) \Gamma(4 q+1)} \\
& +\left(-c_{1}^{10} c_{3}^{3}-c_{1}^{2} c_{3}^{20}-c_{1}^{20} c_{3}^{2}\right) \frac{\Gamma(5 q+1)}{\Gamma(2 q+1) \Gamma(3 q+1)}+20 \operatorname{sign}\left(c_{4}^{40}\right) \\
& c_{2}^{51}=-c_{1}^{0} c_{3}^{41}-c_{1}^{1} c_{3}^{31} \frac{\Gamma(6 q+1)}{\Gamma(q+1) \Gamma(5 q+1)}-c_{1}^{10} c_{3}^{30} \frac{\Gamma(6 q+1)}{\Gamma(2 q+1) \Gamma(4 q+1)}-c_{1}^{20} c_{3}^{20} \frac{\Gamma(6 q+1)}{\Gamma^{2}(3 q+1)}, \\
& c_{3}^{5}=-2.5 c_{3}^{4}+8 c_{1}^{0} c_{1}^{4}+8 c_{1}^{1} c_{1}^{3} \frac{\Gamma(4 q+1)}{\Gamma(q+1) \Gamma(3 q+1)}+4\left(c_{1}^{2}\right)^{2} \frac{\Gamma(4 q+1)}{\Gamma^{2}(2 q+1)}, \\
& c_{3}^{50}=-2.5 c_{3}^{40}+8 c_{1}^{0} c_{1}^{40}+8 c_{1}^{1} c_{1}^{30} \frac{\Gamma(5 q+1)}{\Gamma(q+1) \Gamma(4 q+1)}+\left(8 c_{1}^{10} c_{1}^{3}+8 c_{1}^{2} c_{1}^{20}\right) \frac{\Gamma(5 q+1)}{\Gamma(2 q+1) \Gamma(3 q+1)}, \\
& c_{3}^{51}=-2.5 c_{3}^{41}+8 c_{1}^{10} c_{1}^{30} \frac{\Gamma(6 q+1)}{\Gamma(2 q+1) \Gamma(4 q+1)}+4\left(c_{1}^{20}\right)^{2}(3 q+1), \\
& c_{4}^{5}=-5 c_{2}^{4}, \\
& c_{4}^{50}=-5 c_{2}^{40}, \\
& c_{4}^{51}=-5 c_{2}^{41} .
\end{aligned}
$$

According to equations (21)-(32), the discrete iterative equations can be obtained which are necessary for the fractional-order system implemented on the DSP platform. For hardware design, the block diagram of the working principle is shown in Figure 17.

In the experiment, the YXDSP-F28335 CORE is employed for its powerful computing ability, and the key chip in this board is the float-point DSP TMS320F28335. A low-power 4 channel and 12-bit parallel D/A converter DAC7724 is used to convert the time series generated by DSP. Then, the converted data are sent to an oscilloscope (Agilent DSO-X 3052A) which is used to capture the phase portraits of the system. The flow diagram for DSP implementation is shown in Figure 18.

Firstly, the DSP is initialized, and initial value settings and some preparation before calculation are made. Then, the 
system is iterated according to equations (19)-(30) and the result is pushed in. In the result processing the data is converted into analog signals and sent to an oscilloscope which is used to record phase portraits. After that, the result is pop out from the DSP and replaces the initial value for the next iteration. Iterations continue until the phrase portrait is complete.

Through the DSP platform, the experimental results were obtained as shown in Figure 19. It is consistent with the numerical simulations and the circuit implementation results.

\section{Finite-Time Synchronization of the Fractional-Order System}

6.1. Finite-Time Stability Theory of Fractional-Order System. The synchronization of fractional chaotic systems has great potential in the field of secure communication. With the development of the research, many synchronization methods have been proposed. In [31], a theory of finite-time stability of the fractional-order system is proposed, which can realize the stability of approximation error in finite time, with fast synchronization speed and strong robustness. In this paper, the finite-time stability theory of the fractionalorder system is used to realize system synchronization control.

Theorem 1 (see[31]). Consider a fractional-order system satisfying

$$
x_{a}^{C} D_{t}^{q} \mathbf{x}^{T}=\frac{\Gamma(2)}{\Gamma(2+q)^{a}} D_{t}^{q} \mathbf{x}\left(\mathbf{x}^{q}\right)^{T} \leq-\lambda\left(\mathbf{x x}^{T}\right)^{\alpha}, \quad \alpha<\frac{q+q^{2}}{2}, \lambda>0,
$$

where $0<q<1$, and the states $\mathbf{x}=\left[x_{1}, x_{2}, \ldots, x_{n}\right]$ and $\mathbf{x}^{q}=\left[x_{1}^{q}, x_{2}^{q}, \ldots, x_{n}^{q}\right]$. Then, the state variable $\mathbf{x}$ of the fractional-order system tends to zero in finite-time $t$, and the system is stable:

$$
t=\left[v(0)^{q-(2 \alpha / 1+q)} \frac{\Gamma(1+q-(2 \alpha / 1+q)) \Gamma(1+q)}{\Gamma(1+2 q-(2 \alpha / 1+q)) \lambda \Gamma(2+q)}\right]^{1 / q},
$$

where $v=\mathbf{x}\left(\mathbf{x}^{q}\right)^{T}$.

Lemma 1. When satisfying $a, b<0$ and $0<c<1$, we can get the inequality as follows:

$$
(a+b)^{c} \leq a^{c}+b^{c} .
$$

Let system (4) be the master system, where $q=0.99$, then its slave system is as follows:

$$
\left\{\begin{array}{l}
\frac{\mathrm{d}^{q} x_{1}}{\mathrm{~d} t^{q}}=a\left(y_{1}-x_{1}\right)-u_{1} \\
\frac{\mathrm{d}^{q} y_{1}}{\mathrm{~d} t^{q}}=b x_{1}-k x_{1} z_{1}+d \operatorname{sign}\left(w_{1}\right)+m-u_{2} \\
\frac{\mathrm{d}^{q} z_{1}}{\mathrm{~d} t^{q}}=-c z_{1}+h x_{1}^{2}-u_{3} \\
\frac{\mathrm{d}^{q} w_{1}}{\mathrm{~d} t^{q}}=-g y_{1}-u_{4}
\end{array}\right.
$$

where $u_{1}, u_{2}, u_{3}$, and $u_{4}$ are the controllers based on Theorem 1 .

Then, let $\quad e_{1}=x_{1}-x, e_{2}=y_{1}-y, e_{3}=$ $z_{1}-z$, and $e_{4}=w_{1}-w$ be the errors between master system (4) and slave system (35). Thus, get the error system

$$
\left\{\begin{array}{l}
\frac{\mathrm{d}^{q} e_{1}}{\mathrm{~d} t^{q}}=a e_{2}-a e_{1}-u_{1}, \\
\frac{\mathrm{d}^{q} e_{2}}{\mathrm{~d} t^{q}}=b e_{1}-k\left(x_{1} e_{3}+z e_{1}\right)+d\left(\operatorname{sign}\left(w_{1}\right)-\operatorname{sign}(w)\right)-u_{2}, \\
\frac{\mathrm{d}^{q} e_{3}}{\mathrm{~d} t^{q}}=-c e_{3}+h x_{1} e_{1}+h x e_{1}-u_{3}, \\
\frac{\mathrm{d}^{q} e_{4}}{\mathrm{~d} t^{q}}=-g e_{2}-u_{4} .
\end{array}\right.
$$

The controllers $u_{1}, u_{2}, u_{3}$, and $u_{4}$ can be designed as

$$
\begin{aligned}
& u_{1}=h x_{1} e_{3}+h x e_{3}+\lambda e_{1}^{\beta}, \\
& u_{2}=a e_{1}+b e_{1}-k z e_{1}+d\left(\operatorname{sign}\left(w_{1}\right)-\operatorname{sign}(w)\right)+\lambda e_{2}^{\beta}, \\
& u_{3}=-k x_{1} e_{2}+\lambda e_{3}^{\beta}, \\
& u_{4}=-g e_{2}+\lambda e_{4}^{\beta},
\end{aligned}
$$

where $\lambda$ and $\beta$ are the parameters related to the controllers.

Theorem 2. Adding the controller of equation (36), the fractional-order error system can be stable in a finite-time.

Proof. Based on error system (35) and designed controllers (36), the approximation error can be gotten: 


\begin{tabular}{|c|c|c|c|}
\hline $\begin{array}{c}\text { DSP } \\
\text { (TMS320F28335) }\end{array}$ & $\begin{array}{c}\mathrm{D} / \mathrm{A} \\
(\mathrm{DAC} 7724)\end{array}$ & $y$ series & $\begin{array}{c}\text { Oscilloscope } \\
\text { (Agilent DSO-X 3052A) }\end{array}$ \\
\hline
\end{tabular}

FIGURE 17: Simplified block diagram for DSP implementation of a fractional-order chaotic system.

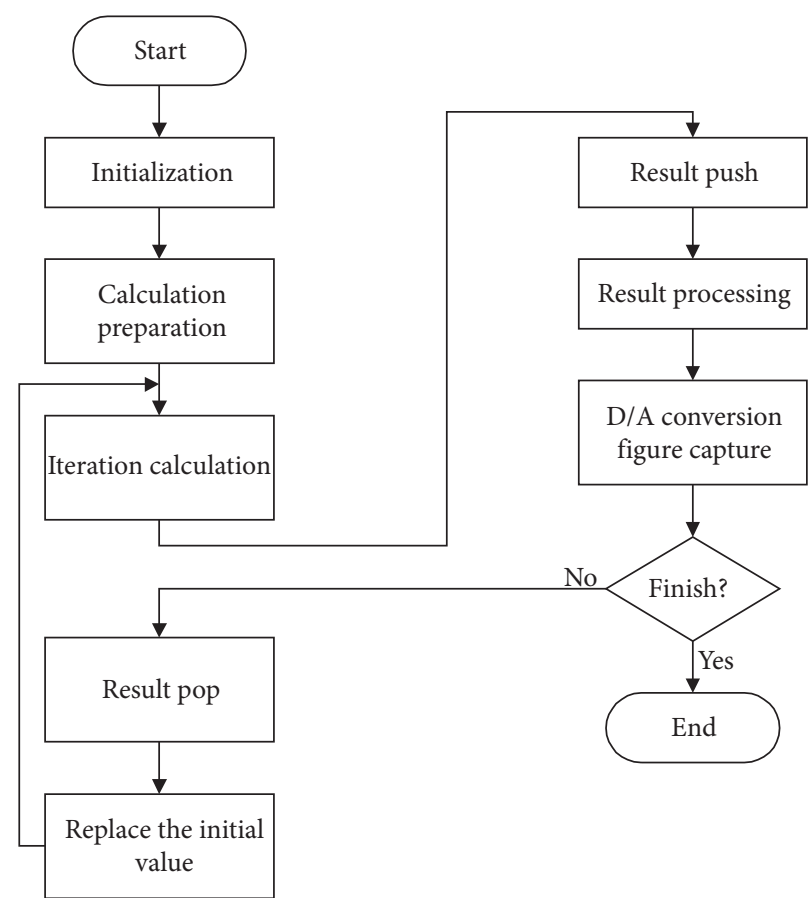

FIGURE 18: Flow diagram for DSP implementation of a fractional-order chaotic system.

$$
\left\{\begin{array}{l}
\frac{\mathrm{d}^{q} e_{1}}{\mathrm{~d} t^{q}}=a e_{2}-a e_{1}-h x_{1} e_{3}-h x e_{3}-\lambda e_{1}^{\beta}, \\
\frac{\mathrm{d}^{q} e_{2}}{\mathrm{~d} t^{q}}=-k x_{1} e_{3}-a e_{1}-\lambda e_{2}^{\beta}, \\
\frac{\mathrm{d}^{q} e_{3}}{\mathrm{~d} t^{q}}=-c e_{3}+h x_{1} e_{1}+h x e_{1}+k x_{1} e_{2}-\lambda e_{3}^{\beta}, \\
\frac{\mathrm{d}^{q} e_{4}}{\mathrm{~d} t^{q}}=-\lambda e_{4}^{\beta} .
\end{array}\right.
$$

According to equation (31), we can get

$$
\begin{aligned}
\frac{\Gamma}{\Gamma(2)}(2) & \frac{\mathrm{d}^{q}}{\mathrm{~d} t^{q}}\left[e_{1} e_{2} e_{3} e_{4}\right]\left[e_{1}^{q} e_{2}^{q} e_{3}^{q} e_{4}^{q}\right]^{T} \\
= & {\left[e_{1} e_{2} e_{3} e_{4}\right]\left[\frac{\mathrm{d}^{q}}{\mathrm{~d} t^{q}} e_{1} \frac{\mathrm{d}^{q}}{\mathrm{~d} t^{q}} e_{2} \frac{\mathrm{d}^{q}}{\mathrm{~d} t^{q}} e_{3} \frac{\mathrm{d}^{q}}{\mathrm{~d} t^{q}} e_{4}\right]^{T} } \\
= & a e_{1} e_{2}-a e_{1}^{2}-h x_{1} e_{1} e_{3}-h x e_{1} e_{3}-\lambda e_{1}^{\beta+1}-k x_{1} e_{2} e_{3} \\
& -a e_{1} e_{2}-\lambda e_{2}^{\beta+1} \\
& -c e_{3}^{2}+h x_{1} e_{1} e_{3}+h x e_{1} e_{3}+k x_{1} e_{2} e_{3}-\lambda e_{3}^{\beta+1}-\lambda e_{4}^{\beta+1} \\
= & -a e_{1}^{2}-c e_{3}^{2}-\lambda e_{1}^{\beta+1}-\lambda e_{2}^{\beta+1}-\lambda e_{3}^{\beta+1}-\lambda e_{4}^{\beta+1} \\
\leq & -\lambda e_{1}^{\beta+1}-\lambda e_{2}^{\beta+1}-\lambda e_{3}^{\beta+1}-\lambda e_{4}^{\beta+1} \\
= & -\lambda\left(e_{1}^{2}\right)^{(\beta+1) / 2}-\lambda\left(e_{2}^{2}\right)^{(\beta+1) / 2}-\lambda\left(e_{3}^{2}\right)^{(\beta+1) / 2}-\lambda\left(e_{4}^{2}\right)^{(\beta+1) / 2} .
\end{aligned}
$$

For the inequality in Lemma 1 , then

$$
\begin{aligned}
& -\left(e_{1}^{2}\right)^{(\beta+1) / 2}-\left(e_{2}^{2}\right)^{(\beta+1) / 2}-\left(e_{3}^{2}\right)^{(\beta+1) / 2}-\left(e_{4}^{2}\right)^{(\beta+1) / 2} \\
& \leq-\left(e_{1}^{2}+e_{2}^{2}+e_{3}^{2}+e_{4}^{2}\right)^{(\beta+1) / 2} \\
& =-\lambda\left(\mathbf{e e}^{T}\right)^{(\beta+1) / 2} .
\end{aligned}
$$

Hence, we can get

$$
\frac{\Gamma(2)}{\Gamma(2+q)} \frac{\mathrm{d}^{q}}{\mathrm{~d} t^{q}}\left[e_{1} e_{2} e_{3} e_{4}\right]\left[e_{1}^{q} e_{2}^{q} e_{3}^{q} e_{4}^{q}\right]^{T} \leq-\lambda\left(\mathbf{e e}^{T}\right)^{(\beta+1) / 2},
$$

where the control parameters are set as $\lambda=1.6$ and $\beta=0.8$. Therefore, according to equation (31),

$$
\alpha=\frac{1+\beta}{2}=0.9<\frac{q+q^{2}}{2}=0.985,
$$

satisfying Theorem 1 . Hence, the master-slave system can realize finite-time synchronization and the error system can also be stable in a finite-time.

6.2. Simulation Results. With $q=0.99$, the time step $t_{\Delta}=0.001$, and final time $T_{\text {sim }}=8 \mathrm{~s}$, the initial conditions of the master system (as equation (4)) and its slave system (as equation $(34))$ are $(2.2,2.4,0.8,0.1)$ and $(3.5,3.4,1.4,-0.5)$, 


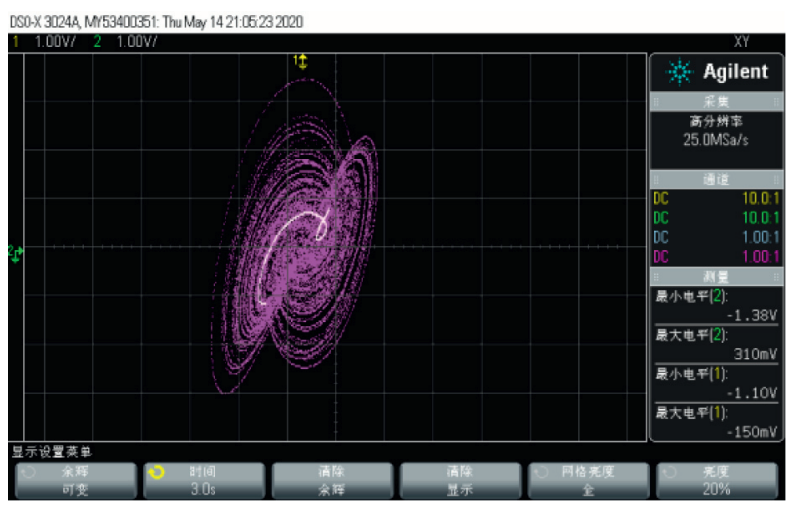

(a)

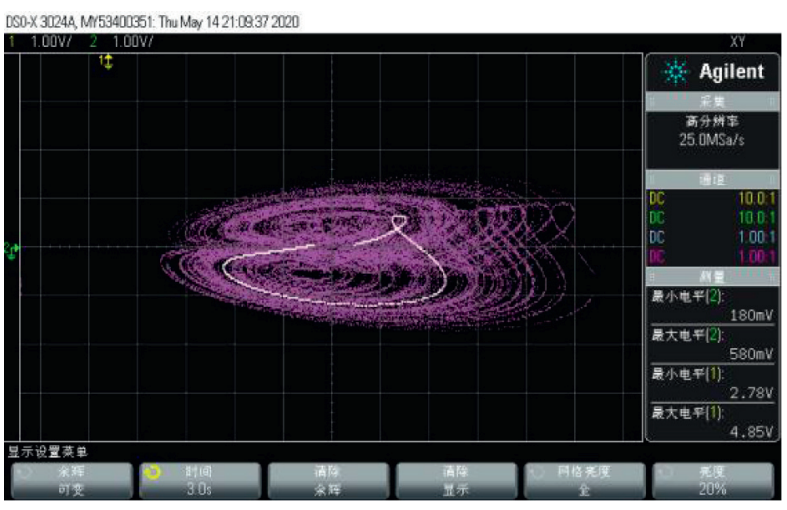

(b)

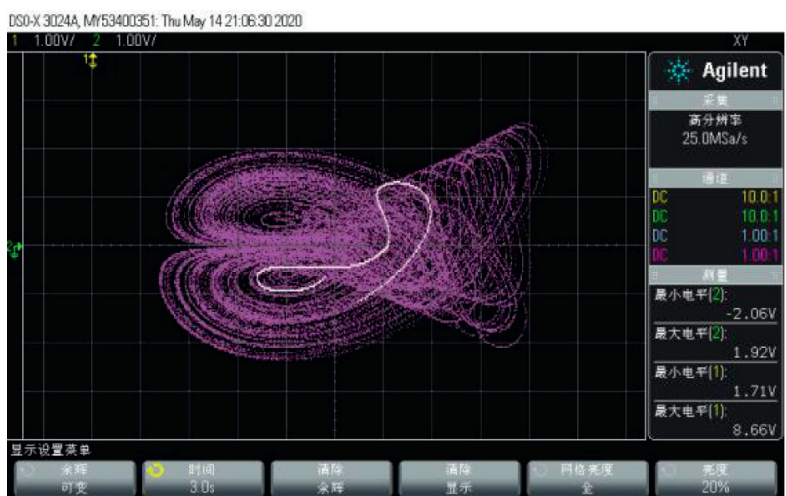

(c)

Figure 19: Experimental attractors of the system using DSP with order $q=0.99$ and parameters $a=10, b=40, c=2.5, k=1, h=4, g=5$, $d=20$, and $m=1$ : (a) on the $x-y$ plane, (b) on the $x-z$ plane, and (c) on the $y$ - $z$ plane.

respectively. Under the control parameters $\lambda=1.6$ and $\beta=0.8$, the simulation result of the chaos synchronization is shown in Figure 20.

If with the initial conditions as $(2.2,2.4,0.8,0.1)$ and (3, $2,1.2,0.5$ ), and the control parameters $\lambda=1.6, \beta=0.8$, the simulation result of the chaos synchronization is shown in Figure 21.

In Figure 20, the synchronization errors $e_{1}, e_{2}, e_{3}$, and $e_{4}$ can convergence to zero in less than 2.5 seconds with the controllers. And the synchronization errors can convergence to zero in less than 2 seconds in Figure 21, for the smaller errors between the initial conditions. Both the results of the two conditions indicate that the state variables of the master-slave system can synchronize in a finite time weather the initial conditions for $w$ have different signs. Therefore, the designed controller is effective.

\section{Conclusion and Perspectives}

This paper has introduced a fractional-order chaotic oscillator based on the Liu system. Changing the parameters, the hidden attractor can have no equilibrium point, in which situation the virtual equilibrium point was studied, and have line equilibrium point. It is worth noting that this infinite number of equilibrium points is not the usual hyperbolic equilibrium point, but the uncommon nonhyperbolic line equilibrium point. In addition, through three-dimensional Poincare, bifurcation, and Lyapunov

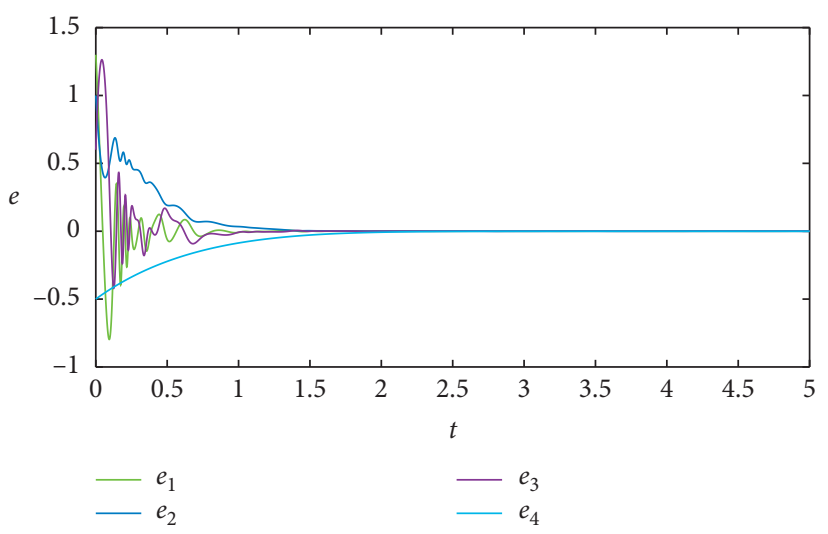

FIGURE 20: Synchronization errors of the states of the system: $e_{1}$ corresponding to the state $x, e_{2}$ corresponding to the state $y, e_{3}$ corresponding to the state $z$, and $e_{4}$ corresponding to the state $w$.

exponential spectrum and the parameter estimation of the system, the influence of the parameters are analyzed. Besides, the feasibility of the new $4 \mathrm{D}$ nonlinear system is confirmed by the help of electronic circuit implementation by means of PSIM and DSP. Finally, based on the theory of fractional finite-time stability, a finite-time synchronization controller is designed to realize the synchronization control of the fractional chaotic 


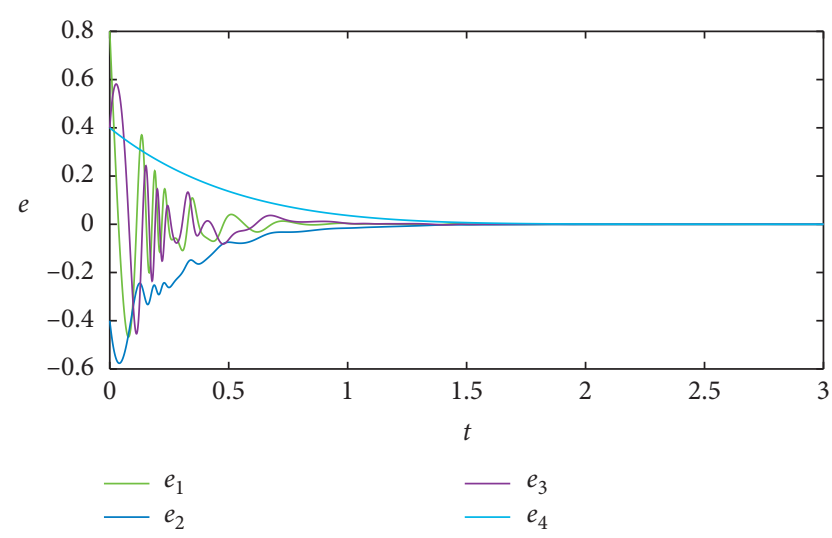

FIgURE 21: Synchronization errors of the states of the system: $e_{1}$ corresponding to the state $x, e_{2}$ corresponding to the state $y, e_{3}$ corresponding to the state $z$, and $e_{4}$ corresponding to the state $w$.

system with hidden attractors. And the validity of the method is verified by numerical simulations. Fractional hidden attractors have important research significance and application value in chaos and cryptography. In the future, we will further explore the application of this research in image encryption technology and secure communication.

\section{Data Availability}

The data used to support the findings of this study are included within the article.

\section{Conflicts of Interest}

There are no conflicts of interest regarding the publication of this paper.

\section{Acknowledgments}

This work was supported by the National Natural Science Foundation of China (grant nos. 51877162 and 51977173).

\section{References}

[1] Z. Hua, F. Jin, B. Xu, and H. Huang, "2D Logistic-Sinecoupling map for image encryption," Signal Processing, vol. 149, pp. p148-161, 2018.

[2] J. Ma, T. Xu, and Y. Hong, "Impact research on a nonlinear cold chain evolutionary game under three various contracts," International Journal of Bifurcation and Chaos, vol. 29, no. 15, Article ID 1950058, 2019.

[3] J. Petrzela, "Fractional-order chaotic memory with wideband constant phase elements," Entropy, vol. 22, no. 4, p. 422, 2020.

[4] Z. Zeng and W. Zheng, "Multistability of neural networks with time-varying delays and concave-convex characteristic," IEEE Transactions on Neural Networks and Learning Systems, vol. 23, no. 2, pp. 293-305, 2012.

[5] Z. Zeng, T. Huang, and W. Zheng, "Multistability of recurrent networks with time-varying delays and the piecewise linear activation function," IEEE Transactions on Neural Networks, vol. 21, no. 8, pp. 1371-1377, 2010.

[6] E. N. Lorenz, "Deterministic nonperiodic flow," Journal of the Atmospheric Sciences, vol. 20, no. 2, pp. 130-141, 1963.
[7] G. Chen and T. Ueta, "Yet another chaotic attractor," International Journal of Bifurcation and Chaos, vol. 9, no. 7, pp. 1465-1466, 1999.

[8] J. Lü and G. Chen, "A new chaotic attractor coined," International Journal of Bifurcation and Chaos, vol. 12, no. 3, pp. 659-661, 2002.

[9] C. Liu, T. Liu, L. Liu, and K. Liu, "A new chaotic attractor," Chaos, Solitons \& Fractals, vol. 22, no. 5, pp. 1031-1038, 2004.

[10] F. Wang and C. Liu, "Studies on Liu chaotic system and its experimental confirmation," Acta Physics Sinica, vol. 55, no. 10, pp. 5061-5069, 2006.

[11] K. Li, J. Cao, and J. He, "Hidden hyperchaotic attractors in a new 4D fractional order system and its synchronization," Chaos, vol. 30, no. 3, Article ID 033129, 2020.

[12] Y. Yu, M. Shi, H. Kang, M. Chen, and B. Bao, "Hidden dynamics in a fractional-order memristive Hindmarsh-Rose model," Nonlinear Dynamics, vol. 100, no. 1, pp. 891-906, 2020.

[13] J. Lu and C. Liu, "Realization of fractional-order Liu chaotic system by circuit," Chinese Physics, vol. 16, no. 6, pp. 15861590, 2007.

[14] X. Wang and M. Wang, "Dynamic analysis of the fractionalorder Liu system and its synchronization," Chaos, vol. 17, no. 3, Article ID 033106, 2007.

[15] A. S. Hegazi, E. Ahmed, and A. E. Matouk, "On chaos control and synchronization of the commensurate fractional order Liu system," Communications in Nonlinear Science and Numerical Simulation, vol. 18, no. 5, pp. 1193-1202, 2013.

[16] Q. Han, C. Liu, L. Sun, and D. Zhu, "A fractional order hyperchaotic system derived from a Liu system and its circuit realization," Chinese Physics, vol. 22, no. 2, Article ID 020502, 2013.

[17] C. Li, J. Sprott, and H. Xing, "Hypogenetic chaotic jerk flows," Physics Letters A, vol. 380, no. 11-12, pp. 1172-1177, 2016.

[18] J. P. Singh and B. K. Roy, "The nature of Lyapunov exponents is $(+,+,-,-)$. Is it a hyperchaotic system?" Chaos, Solitons \& Fractals, vol. 92, pp. 73-85, 2016.

[19] S. Jafari, J. Sprott, V. Pham, S. Golpayegani, and A. Jafari, “A new cost function for parameter estimation of chaotic systems using return maps as fingerprints," International Journal of Bifurcation and Chaos, vol. 24, no. 10, Article ID 1450134, 2014.

[20] W. Xue, Y. Li, S. Cang, H. Jia, and Z. Wang, "Chaotic behavior and circuit implementation of a fractional-order permanent magnet synchronous motor model," Journal of the Franklin Institute, vol. 352, no. 7, pp. 2887-2898, 2015.

[21] Z. Wang, H. Tang, and Z. Chen, "The design and implementation of a multi-wing chaotic attractor based on a fiveterm three-dimension system," International Journal of Circuit Theory and Applications, vol. 44, no. 5, pp. 1186-1201, 2016.

[22] S. Wang, S. Lao, H. Chen, J. Chen, and S. Chen, "Implementation of the fractional-order Chen-Lee system by electronic circuit," International Journal of Bifurcation and Chaos, vol. 23, no. 2, Article ID 1350030, 2013.

[23] S. Zhang, Y. Zeng, Z. Li, and C. Zhou, "Hidden extreme multistability, antimonotonicity and offset boosting control in a novel fractional-order hyperchaotic system wthout equilibrium," International Journal of Bifurcation and Chaos, vol. 28, no. 13, Article ID 1850167, 2018.

[24] M. Wang, X. Liao, Y. Deng, Z. Li, Y. Zeng, and M. Ma, "Bursting, dynamics, and circuit implementation of a new fractional-order chaotic system with coexisting hidden 
attractors," Journal of Computational and Nonlinear Dynamics, vol. 14, no. 7, Article ID 071002, 2019.

[25] A. Charef, H. H. Sun, Y. Y. Tsao, and B. Onaral, "Fractal System as represented by singularity function," IEEE Transactions on Automatic Control, vol. 37, no. 9, pp. 1465-1470, 1992.

[26] A. Akgul, H. Calgan, I. Koyuncu, I. Pehlivan, and A. Istanbullu, "Chaos-based engineering applications with a 3D chaotic system without equilibrium points," Nonlinear Dynamics, vol. 84, no. 2, pp. 481-495, 2016.

[27] G. Adomian, "A new approach to nonlinear partial differential equations," Journal of Mathematical Analysis and Applications, vol. 102, no. 2, pp. 420-434, 1984.

[28] D. Cafagna and G. Grassi, "Bifurcation and chaos in the fractional-order Chen system via a time-domain approach," International Journal of Bifurcation and Chaos, vol. 18, no. 7, pp. 1845-1863, 2018.

[29] N. Shawagfeh, "Analytical approximate solutions for nonlinear fractional differential equations," Applied Mathematics and Computation, vol. 131, no. 2-3, pp. 517-529, 2002.

[30] S. He, K. Sun, and S. Banerjee, "Dynamical properties and complexity in fractional-order diffusionless Lorenz system," European Physical Journal Plus, vol. 131, no. 8, pp. 254-2016.

[31] L. Zhao, J. Hu, and X. Liu, "Adaptive tracking control and synchronization of fractional hyper-chaotic Lorenz system with unknown parameters," Acta Physics Sinica, vol. 59, no. 4, pp. 2305-2309, 2010. 